\title{
MULTIMODAL AUTOMATED LAST-MILE DELIVERY SYSTEM: DESIGN AND APPLICATION
}

by

Farah Samouh

Bachelor of Science in Civil Engineering, The University of Jordan, 2017

\author{
A thesis \\ presented to Ryerson University \\ in partial fulfillment of the \\ requirements for the degree of Master of Science \\ in the program of Civil Engineering \\ Toronto, Ontario, Canada, 2019 \\ (C) Farah Samouh, 2019
}




\section{Author's Declaration}

I hereby declare that I am the sole author of this thesis. This is a true copy of the thesis, including any required final revisions, as accepted by my examiners.

I authorize Ryerson University to lend this thesis to other institutions or individuals for the purpose of scholarly research.

I further authorize Ryerson University to reproduce this thesis by photocopying or by other means, in total or in part, at the request of other institutions or individuals for the purpose of scholarly research.

I understand that my thesis may be made electronically available to the public. 


\begin{abstract}
Multimodal Automated Last Mile Delivery System: Design and application

By

Farah Samouh

MASc. in Civil Engineering - Department of Civil Engineering

Ryerson University

2019

This thesis focuses on exploring the emerging automated technologies for last-mile on-demand food delivery as a new means of transportation to reduce congestion in urban areas. In order to achieve that 4 systems are designed and evaluated: Robot delivery system, drone delivery system and two hybrid delivery systems. Both hybrid systems are based on hub-spoke networks, Hybrid System 1.0 uses robots for phase one of the delivery and drones for phase two Hybrid System 2.0 uses drones for phase one and robots for phase two. To evaluate the efficiency of these systems, an in-house agent-based simulation model in MATLAB is developed for the City of Mississauga. 30 scenarios are tested differing in terms of demand and fleet size. The results show that Hybrid system 2.0 is the most efficient system of all four proposed due to the use of hub, customer waiting
\end{abstract} time and landing zones for drones. 


\section{Acknowledgments}

Thank you God Almighty for giving me the strength, knowledge, ability and opportunity to undertake this research study. Without your blessings, this achievement would not have been possible.

During my masters journey, I was lucky to be mentored by my thesis advisor Dr.Bilal Farooq. The door to his office was always open, whenever I was faced with troubles during my research or had any questions, I could reach him easily for help. He allowed this thesis to be my own work but steered me in the right direction whenever he believed I needed it. Thank you for the opportunities you have given me that allowed me to grow.

Throughout the writing of this dissertation I have received a great deal of support, assistance and collaboration from my teammates at the laboratory of innovation in transportation at Ryerson University. In particular, thank you to Dr.Shadi Djavadian, Veronica Gluza and S.Mehdi Meshkani who provided me with help and support throughout my masters and especially during the submission of my journal paper.

My acknowledgement would be incomplete without thanking the biggest source of my strength, my family. Thank you to my mother who is a transportation engineer herself. She is my Idol who has always supported me during the tough times I have faced during my masters. To my father whom always believed in me and pushed me to become the person I am today. And to my siblings; Dana, Jamil, Malik and Waleed for being the emotional support I needed at times when I was overwhelmed, they made sure I pushed through until I reached this day. Finally, a deep thank you to my aunts and uncles who are miles away but have been unconditionally supportive during my master's journey. Being the oldest granddaughter, I hope to set an example of persistence and hard work for all my younger cousins. You are capable of achieving greatness just believe in yourself.

And to my amazing friends from all over the world, this would not have been possible without their unselfish love and support given to me at all times. I would like to thank Shubham Bohra, Niraj Das and Fakruddin Syed, who were always a call away and ready to help with whatever I needed to ensure I reached my goals.

The blessings of my late grandfather have kept me on the right path. His continuous encouragement throughout my years of study was an instrumental key to my success.

This one is for you Grandpa! May I always make you proud of the person I have become today. 


\section{Table of Contents}

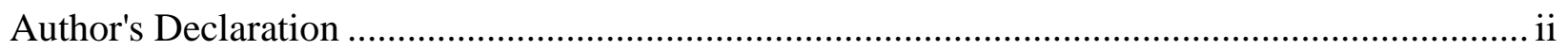

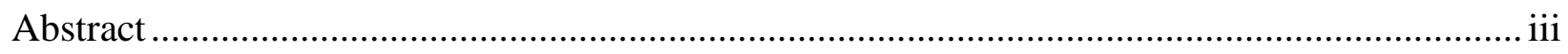

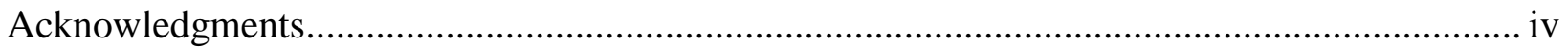

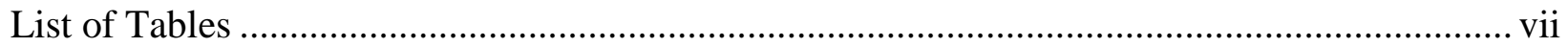

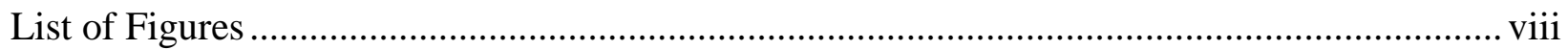

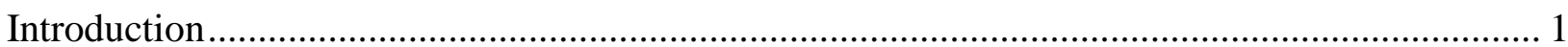

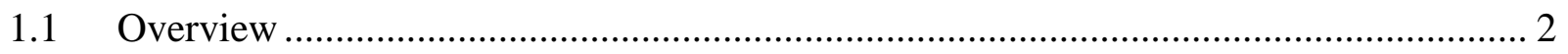

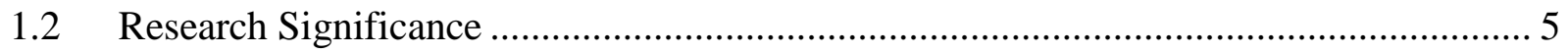

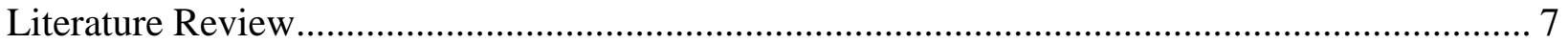

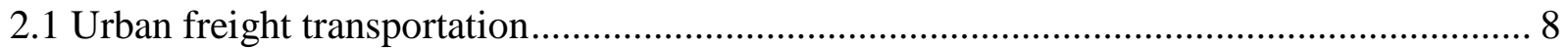

2.1.1 The effect of urban freight transportation on congestion......................................... 9

2.2 On-demand last mile delivery services using autonomous technologies............................. 10

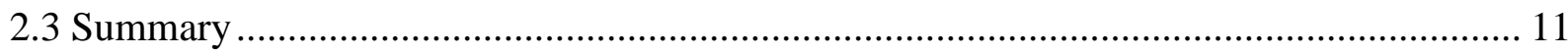

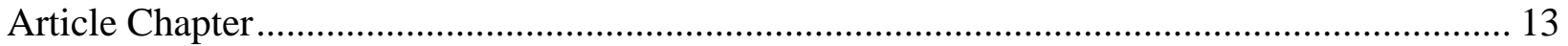

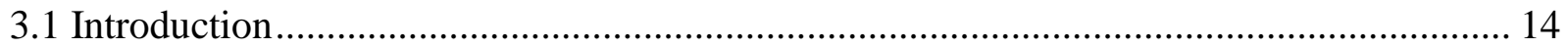

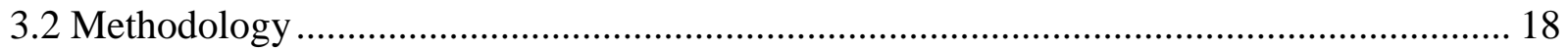

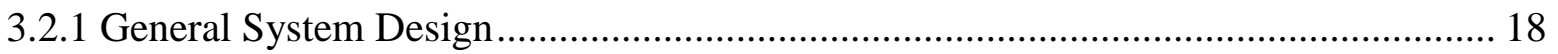

3.2.2 Proposed delivery process for delivery robots and drone systems ............................. 19

3.2.3 Proposed delivery process for hybrid delivery system ................................................ 24

3.2.4 Performance criteria for all delivery systems .......................................................... 28

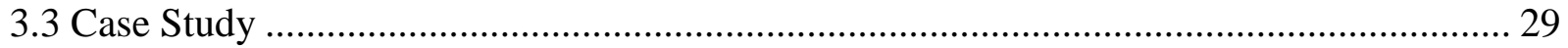

3.3.1 Food Delivery Systems Operating Policy Simulation ............................................... 33

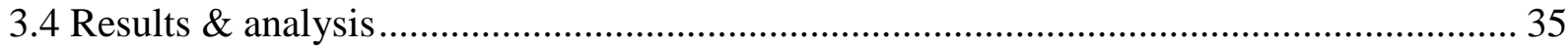

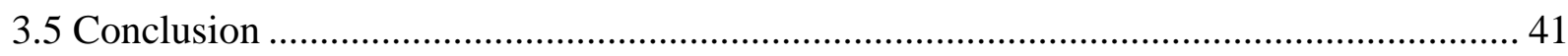

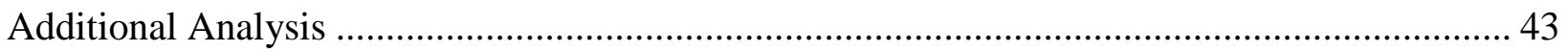

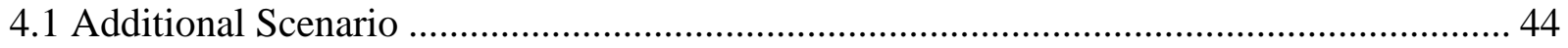

4.1.1 Proposed delivery process for hybrid delivery system ............................................... 44

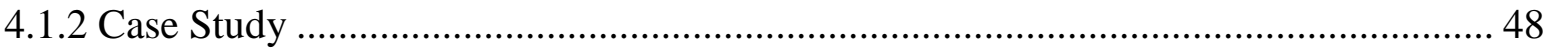

4.1.3 Results \& Analysis............................................................................................... 50

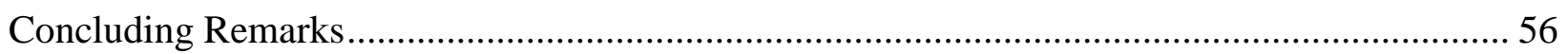


5.1 Conclusion .

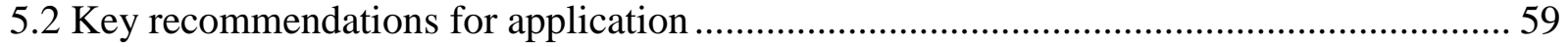

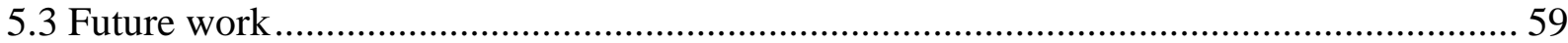

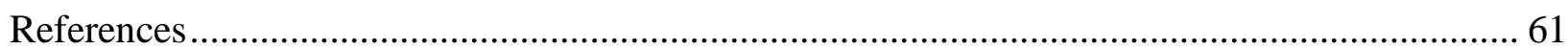




\section{List of Tables}

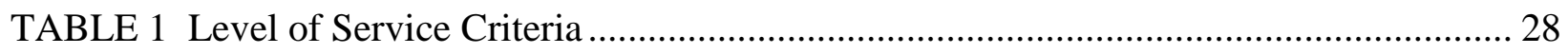

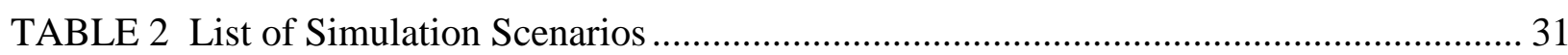

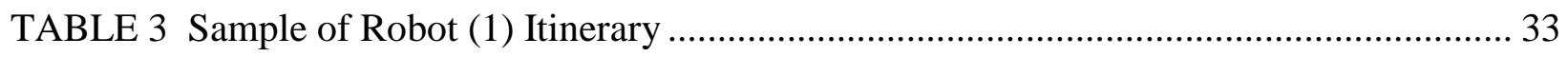

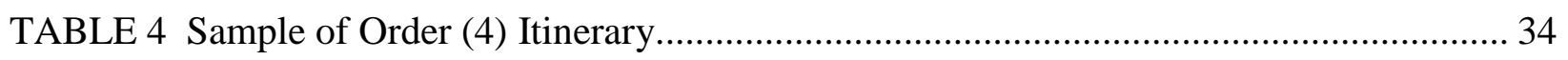

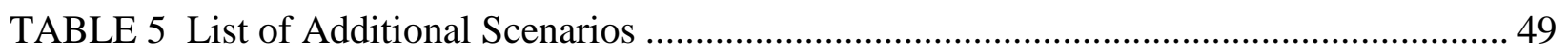




\section{List of Figures}

FIGURE 1 : Users market penetration rates of online food delivery in Canada ......................... 4

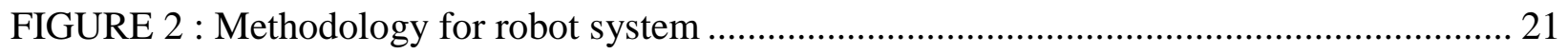

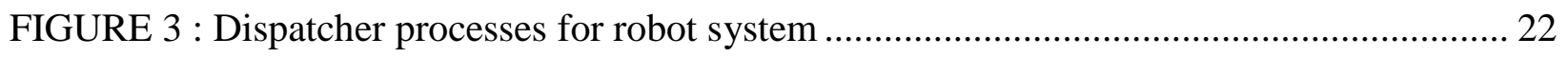

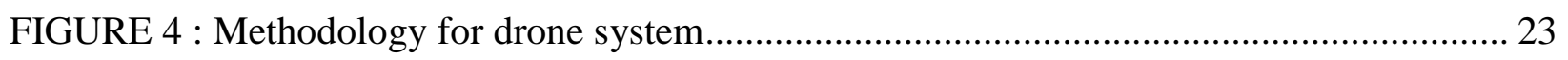

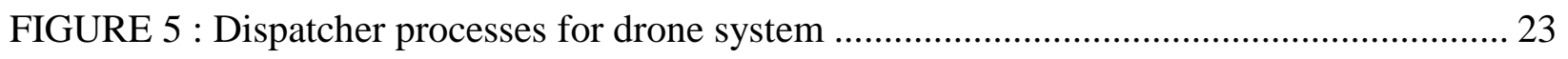

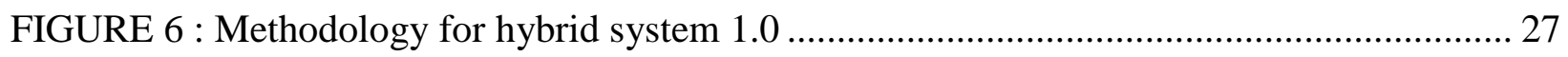

FIGURE 7 : Dispatcher processes for hybrid system 1.0 ................................................... 27

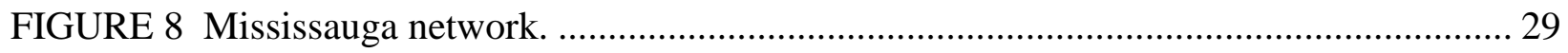

FIGURE 9: Average customer wait time for robots system. ............................................. 36

FIGURE 10 : Average customer wait time for drones system. ........................................... 37

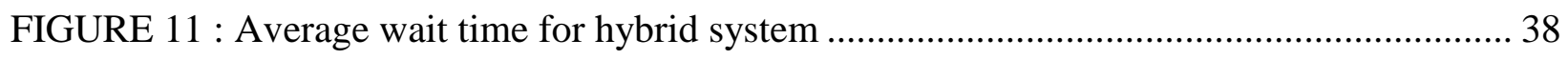

FIGURE 12 : Level of service for scenario 25 robots - 10 drones in the hybrid system............. 39

FIGURE 13 : Average wait time for optimal starting fleet sizes per system .......................... 40

FIGURE 14 : Methodology of hybrid system 2.0............................................................ 46

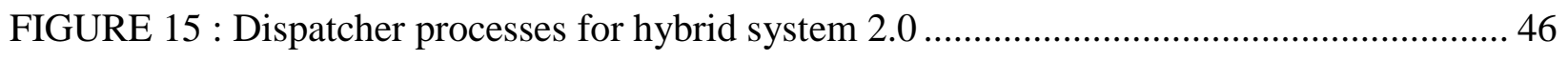

FIGURE 16 : Detailed network for scenario 4: (a) drones network - (b) robots network ........... 48

FIGURE 17 : Average wait time for hybrid system 2.0 ..................................................... 51

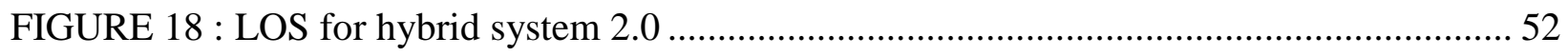

FIGURE 19 : Average wait time for optimal scenarios for hybrid system 2.0 ...................... 53

FIGURE 20 : Average wait time for optimal starting fleet sizes for all four systems................ 54 
Chapter 1

\section{Introduction}




\subsection{Overview}

Urban freight transportation and logistics operations are concerned with the activities related to delivering and collecting goods in urban areas. These activities are often referred to as city logistics as they involve the processes of transportation, handling, and storage of goods, the management of inventory, waste, and returns as well as home delivery services [1]. While urban freight transportation increases the economy and provides thousands of jobs, these services come with an expensive cost by adding congestions and impacting the environment [2]. 55\% of the world's population lives in urban areas nowadays and it's expected to increase to $68 \%$ by 2050 with the most urbanized region being Northern America, with $82 \%$ of its population living in urban areas in 2018 [3].

As Barbosa et al. mentioned "City Logistics" is an important domain of urban freight transportation. With more people living implies higher demand for transportation services are needed which will cause a significant increase in congestion unless addressed properly[4] .Congestion caused by urban freight transportation could be due to several reasons e.g. conflict between freight and passenger services, increase in e-commerce and one-day delivery services, restrictions on the operational hours of freight movement in urban areas and parking limitations within congested areas, where trucks occupy curb space, and often double park in bicycle lanes and parts of traffic lanes [5]. The Federal Highway Administration estimated 947,000 hours annually of vehicle delay attributed to delivery trucks parked at curbside in dense urban areas due to the limited existence of proper loading and unloading parking spaces [6]. 
In Canada, most passengers and goods travel by road, where $72 \%$ of produced goods that remained in Canada were transported via trucking ${ }^{1}$ services in 2015 . Trucking companies were concentrated in four provinces Ontario being the highest at (41.1\%) followed by Alberta (16.3\%), Québec (15.1\%), and British Columbia (14.6\%) [7].

On-demand transportation services are the transportation of people or goods upon request from a user[8] . The rise of these services such as ridesharing and food delivery apps have led to a significant increase in congestion in urban areas [9]. A study conducted by Transportation consultancy Fehr \& Peers indicated that Uber and Lyft make up as much as 13.4 percent of all vehicle $^{2}$ miles in San Francisco County. In Boston, it's 8 percent; in Washington, DC, it's 7.2 percent [9].

Moreover, Online food delivery is considered a part of urban logistic when it comes to food delivery within urban areas. In Canada, in the online food delivery service revenue is expected to show an annual growth rate of $10.2 \%$, resulting in a market volume of US $\$ 2,959 \mathrm{~m}$ by 2023 , and it is expected to reach a total users market penetration rate of $39.4 \%$ in 2023 as demonstrated in figure $1[10]$.

\footnotetext{
${ }^{1}$ Trucking includes many small for-hire carriers and owner operators, and some medium and large for-hire companies that operate fleets of trucks and offer logistic services.

${ }^{2}$ All other passenger and freight vehicles
} 


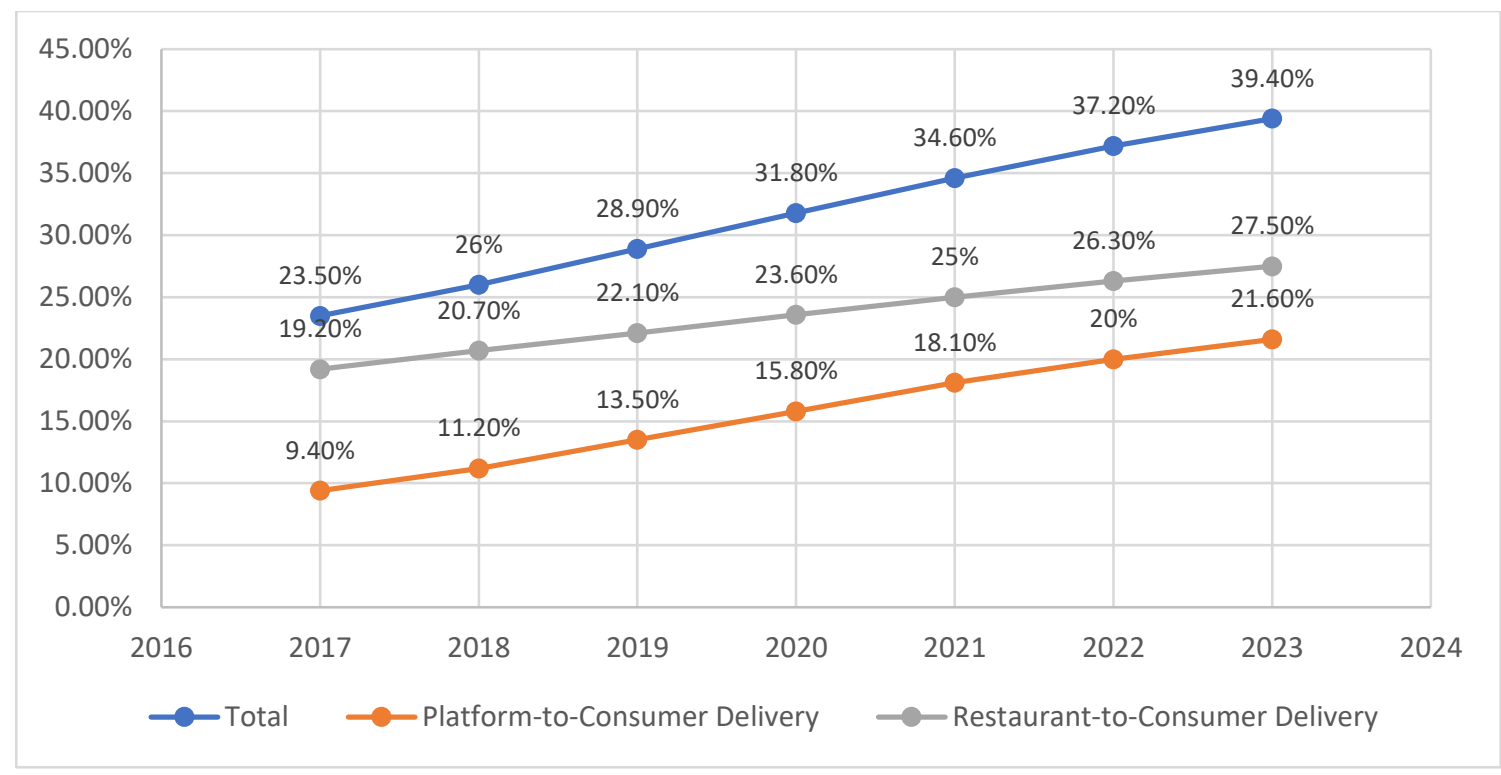

FIGURE 1 : Users market penetration rates of online food delivery in Canada

Furthermore, In Canada UberEATS is planning to expand their deliveries beyond restaurant deliveries to include groceries, meal kits and restaurant supplies delivery [11] which is expected to result in adding more congestion in the near future.

These challenges are being mitigated and addressed by looking into off-peak delivery hours, Freight Consolidation, Central delivery stops and Innovative Technologies [5]. In Canada, Peel Region Launched an Off-Peak Delivery Pilot project to try and mitigate the effects of Traffic congestion on societies and industries across Ontario, especially within the Greater Toronto and Hamilton Area (GTHA) [12]. Furthermore, central pick-up centers have been established such as Doodle in London offering customers the option for their packages to be delivered at their centers rather than homes allowing the customer to pick-up their packages at their own convenience [13]. Also, US created centralized drop-off lockers through providing a central location for customers to pick up their items without the need of staff for such services reducing vehicle dwell time, where 
the vehicle only needs to stop once and not at every home and eliminating failed first time deliveries [14].

Last but not least, emerging autonomous transportation technologies can be integrated with existing systems to address transportation challenges while their applications can be used to reduce congestion, increase safety levels, reduce green gas house emissions and sustain economic vitality. Last mile delivery oversees delivering the product or service to its final destination. Autonomous Ground Vehicles (AGVs) and Autonomous Aerial Vehicles (AAVs) are being used for last-mile delivery services worldwide.

This dissertation argues the need to take a pro-active approach into integrating new emerging technologies such as robots and drones via different systems for last mile on-demand food delivery applications as an initiative to reduce congestion in dense urban areas while maintaining an acceptable customer wait time. Food delivery systems operate in a similar manner as ridesharing systems except there are no options for carpooling which means every order gets assigned to a specific driver and their vehicle. However, in this dissertation we evaluated four different delivery systems that use autonomous technologies that operate either on the sidewalks or flying through air as a substitute for vehicles.

\subsection{Research Significance}

The performance of transportation systems is of crucial importance for individual mobility, commerce, and for the economic growth of urban areas. In this context, urban freight transportation is an essential contributing activity for that growth. As when it comes to the last mile urban freight transportation there has been efforts to measure and control freight movement within highly congested areas using conventional methods. However, with new technologies emerging this 
research will design, operate and investigate the effect of incorporating autonomous technologies such as drones and robots as a means for last mile urban freight transportation. What are the effects of deploying various automaniac technologies for last mile delivery in terms of reducing order wait time in urban areas?

This research will be investigating the operational designs and efficiency by applying AGVs and AAVs for last mile food delivery services within a highly populated urban area in three systems: Robot system, Drone system, and Hybrid system. Research significance can be outlined as follows:

- This research will help companies comprehend the benefits of using intelligent transportation to maximize their profit and being able to reduce delivery times

- As most of the existing models focus on designing solutions for improvement of the last mile urban freight transportation using freight consolidation, this research will study the effect of adding new technology advances to help better improve the existing system

- By conducting a detailed analysis in a highly populated urban area and incorporating autonomous technologies will lead to more realistic insights on how to improve deliveries

- This research will help engineers and planners improve city planning by preparing for the connected and autonomous technologies. Allowing these technologies to be used for commercial purposes will result in increased economic competitions while reducing delays and increasing safety on our roads. 


\section{Chapter 2}

\section{Literature Review}




\subsection{Urban freight transportation}

While the pace of growth for overall retail sales is subdued, the digital portion of sales continues to expand rapidly. Global retail e-commerce sales which include products and services (barring travel, restaurant, and event ticket sales) ordered via the internet over any device are projected to reach $\$ 27$ trillion by 2020 . In 2014 Canada was ranked the $8^{\text {th }}$ largest e-commerce market worldwide with sales increasing from 2013 by $17.4 \%$ and reaching $\$ 24.63$ billion $^{3}$ in 2014 . Furthermore, it was found that e-commerce sales as a percent of the total retail sales in Canada were 5.2\% with Amazon being the top online retailer [15].

With this rapid increase in e-commerce and companies wanting to expand their businesses online, there will be a growth in home delivery causing a significant impact on urban freight transportation. This impact will rise as internet shopping is intertwined with express delivery to homes or other appointed destinations which will lead to more trucks, cars on the streets causing congestion and an increase in greenhouse gas emissions [16].

Maintaining cost efficiency and a high level of service in the distribution system is crucial for the logistics service providers to remain competitive in the online business [17]. The challenge lies in how logistics service providers should react to accommodate the increase in demand triggered by the rapid growth of an online market to build an effective last mile freight delivery system.

Last mile delivery is defined as "the movement of goods from a transportation hub to the final delivery destination"[18]. Last mile delivery is considered an important aspect of urban freight transportation since it includes making deliveries to personal residence, retail stores, restaurants,

\footnotetext{
${ }^{3}$ Travel websites and event tickets are not included as part of retail numbers.
} 
and other merchants in a central business district which often contributes to congestion and safety problems in urban areas [19].

Freight delivery is the transportation of good or services without specifying a certain area, but as we specify the term by using the word urban ${ }^{4}$, it solely focuses on investigating the activities of delivering and collecting goods and services in town and city centers that contain a high population density. Freight movement regional models are performed on a larger scale integrating rail, air and ship transport however, same models cannot be implied on urban freight transport due to the difference characteristics where most freight transport in urban areas is carried on the roads. Furthermore, Urban freight transportation models cannot be developed based on large truck commercial trips thus neglecting light vehicle commercial trips.

\subsubsection{The effect of urban freight transportation on congestion}

Stefan et al.[21] estimated that approximately $15 \%$ of vehicle movement in urban areas is due to commercial vehicles. They differentiated urban commercial movement from regional commercial movement and household movement therefore developing a tour-based microsimulation of urban commercial vehicle movements (UCVM) for the city of Calgary, Alberta.

Moreover, Ferguson et al.[22] introduced a hybrid microsimulation framework based on a disaggregate and behavioral tour-based simulator and aggregate zonal trip generation and distribution modules estimating the travel pattern of heavy, medium and light commercial vehicles

\footnotetext{
${ }^{4}$ According to Statistics Canada (StatCan), an urban area in Canada is an area with a population of at least 1,000 people where the density is no fewer than 400 persons per square kilometer (1,000 per square mile) [20].
} 
in the Greater Toronto and Hamilton Area (GTHA). The model proposed is considered an estimate model providing "ball park” estimates of commercial vehicle movements.

With the increase in e-commerce and the existence of ridesharing services Qi et al. [23]studied the ability for using crowdsourced shared mobility for delivery services on a large scale for retail ecommerce.. while there are companies that started implementing crowdsourced delivery such as UberEATS and Amazon Flex, however, these companies focus on express deliveries on a smaller scale.

As a solution to reduce congestion in urban areas Iwan et al.[24]conducted an investigation to analyze the usability and efficiency of parcel lockers as a last mile delivery solution. It was found that the most important factor of efficiency for parcel lockers solution is having the appropriate location for the lockers used for deliveries.

\subsection{On-demand last mile delivery services using autonomous technologies}

On-demand transportation services are "a form of transport service where vehicles alter their routes based on particular transport demand rather than using a fixed route or timetable [25]." These services provide pick-up and drop-off to passengers and good upon a person's request.

Meanwhile, technology has fundamentally transformed the way industries such as music, books and video operate and the way our delivery systems are built is no exception, as traditional restaurants are expanding their digital presence to accommodate for that change by providing online purchases and delivering them to the consumer. Nowadays, on-demand services such as Uber has expanded their services to not only connect drivers of personal vehicles with passengers but to connect drivers for food delivery pick-ups as well. 
For the application of drones, Dorling et al.[26]proposed solutions for two multi-trip vehicle routing problems for drone delivery by applying simulated annealing heuristic to minimize costs depending on a delivery time limit and to minimize the overall delivery time bound by a budget constraint. Furthermore, Ha et al. [27]. modified the travelling salesman problem (TSP) to drones naming it TSP with drone (TSP-D) to minimize operational costs including total transportation cost and the minimum time.

Murray and Chu [28] investigated the routing problem related to the truck-drone combination for delivery calling it the Flying Sidekick Traveling Salesman Problem (FSTSP) which is a new variant of the traditional traveling salesman problem (TSP) for drones working in tandem with a delivery truck to minimize total delivery time. Ferrandez et al. [29]followed Marray and Chu's research in order to develop an operational delivery system that maximize deliveries while minimizing time and energy based on K-means and Generic Algorithm.

In parallel to the research completed regarding the commercial use of drones for delivery purposes, such research has been conducted in the realm of deploying robots for commercial use although such robots exist and are currently operational. One research was conducted by Yuan and Gong studied the use of robots in transporting movables shelves to order pickers in a system called robotic mobile fulfillment system (RMFS) [30]. However, this system involves the movement of goods in a warehouse environment thus not facing the routing challenges that apply to robots navigating the streets.

\subsection{Summary}

With the integration of Autonomous technologies for last-mile on-demand delivery it is becoming vital to understand and predict the effectiveness of incorporating these disruptive mobility 
technologies for the reduction in congestion. This chapter outlined the background theory and existing research in the context of this thesis. After conducting an extensive literature review, to the best of our knowledge no food delivery system has been fully designed and applied using autonomous technologies. In this dissertation four on-demand last-mile food delivery systems were proposed and evaluated by implementing autonomous vehicles (drones and robots). 


\section{Chapter 3}

\section{Article Chapter}

"Simulation Based Design and Application of Multimodal Automated Last-Mile Delivery System"

*** Under-review in the journal of Transportation Research Record 


\subsection{Introduction}

Travel demand in urban areas continues to grow resulting in increased congestion on the existing networks. As the population increases and the economy grows, car ownership continues to rise. For example, in Canada the number of vehicles registered increased by 1.8\% from 2015 to 2016 and continued to increase by $1.6 \%$ from 2016 reaching 34.3 million vehicles in 2017 [31][32]. With this rate of increase, the existing infrastructure will not be able to keep up with the rise in demand and in fact, it is neither sustainable nor realistic to build enough roads and infrastructure to comfortably accommodate this increase. Therefore, there has been an ongoing movement across the globe to try and find solutions that are practical and feasible in terms of adapting to the growth in travel demand.

Parallel to that, on-demand services have shown large growth in recent years. A study by Castiglione, Cooper, Sana, Tischler and Chang [33] has been conducted to evaluate and quantify the causes of congestion in San Francisco between 2010 and 2016 after congestion worsened. It was found that on-demand transportation companies such as Uber and Lyft are primary factors in contributing to the congestion. The effect of these services varies through the day, having the highest affect during evening peak hours, accounting for $70 \%$ of the increase in vehicle delay.

Adding to that in particular, food delivery Apps are reshaping how we purchase food. With the advancements in information and communication technologies, the traditional approach to ordering food over the phone and waiting for it to be delivered has been transformed into ordering digitally through a smartphone Apps and tracking one's order through the whole process up until the point of delivery or maybe even ordering and getting notified when the order is ready for pickup if one chose that option. These services have their perks and have undeniably provided efficient on-demand last mile transportation services. Nevertheless, they are a major factor that contributes 
to the rise in congestion in urban areas. Therefore, this study explores solutions that can provide the same services, but without impacting the existing congestion on our road networks. Smart transportation models are emerging globally and automating last mile delivery services is being investigated to cope with the high increase in demand. By using the word automation two possibilities come in place; the use of autonomous ground vehicles (AGV's) and Autonomous aerial vehicles as a mean of transportation resulting in further redefining the traditional delivery services.

Autonomous ground vehicles (AGV's) is a generic term that represents autonomous cars that drive on the paved road or autonomous robots that operate on the sidewalk depending on the size, weight, and distance that the parcel needs to be delivered to. AGVs with parcel lockers will substitute current forms of regular parcel delivery, as current companies are testing this new delivery services of transporting goods for last mile delivery to provide a new level of convenience, and to reduce cost on the consumer but correspondingly increase companies' revenue [34]. The other type of AGV's that can be used are small autonomous vehicles that deliver parcels to the doorstep. These vehicles are relatively slower and use the sidewalk rather than the street to reach their destination. Hoffmann and Prause [35] discussed the regulatory framework for last mile-delivery robots by foreseeing the legal challenges these delivery robots will bring to the surface. However, no real case study was incorporated to systematically see the effect of applying these technologies on delivery timings or congestion and traffic.

On the other hand, and with the recent developments in technology, unnamed aerial vehicles or “drones" are being investigated for their potential to bring many changes in the way businesses and government agencies operate. Previously drones were being used mostly for military surveillance and tactical applications. However, currently, a wide range of applications for the use 
of drones in recreational and commercial purposes are being investigated. Drones are being utilized to transport packages, food or other goods. For example, drones can be used to provide health care services, postal delivery services as well as food delivery services.

Drones and robots are considered emerging technologies in the field of transportation and are defying the orthodox delivery systems currently used. Package companies and logistics firms are looking for the best options for that final step of a product's journey also known as last-mile, and an increasing number of companies are aiming to use robots and aerial drones to deliver goods to customers. McKinsey has predicted that autonomous vehicles including drones will deliver up to 80 percent of all items by 2025 [36].

Researchers have been exploring different aspects of drone delivery regarding its costeffectiveness and impact on the environment but it's a fairly new research topic when it comes to autonomous deliveries. A study conducted by Jung and Kim [37] focused on analyzing specifications of amazon delivery drones and the delivery cost that accompanied with this new change, in using drones for the delivery. Though, delivery time and environmental impacts on drone delivery weren't considered in this research.

However, Goodchild and Toy [38] evaluated the effect of unmanned aerial vehicle technology in reducing $\mathrm{CO} 2$ emissions in the delivery service industry. The study concluded that net emissions differences can fluctuate vastly across delivery service zones, as they are profoundly influenced by the number of recipients and distance away from their take-off location. Yet, a life cycle assessment (LCA) should be pursued to acquire a more holistic perspective on environmental impacts. 
The compelling need for service innovation has led to the development of this study. As demonstrated above each technology has been applied for use across various services. Nevertheless, these food delivery services share many similarities to customer transportation services. However, to the best of our knowledge there has not been any study evaluating the use of robots, drones, and their hybrid by a third-party delivery service. The premise is that these emerging technologies have the capability to relief congestion by taking on-demand delivery trips off the road network as well as delivery cost, while maintaining an equivalent or better service delivery times.

We propose a fully integrated delivery system for the on-demand last-mile delivery services that employ various automation technologies (ground and aerial) and is focused on taking the additional trips generated by such services off the already congested urban roads. Three delivery systems are investigated that are based on: a) robot technology based delivery system b) drone technology based delivery system and c) a hybrid of the two. A detailed analysis of system's operation and the integration of autonomous technologies in the delivery system is conducted in a real case study of city of Mississauga, Canada - a densely populated urban area. The case study focuses on the on-demand food delivery problem. The purpose of this study is multifold:

1- It investigates the effectiveness of adding new autonomous technologies separately and combined to the on-demand food delivery system by maintaining a high level of service in terms of delivery times

2- It investigates three different delivery models and compare them to determine the most efficient model in terms of minimum delivery times.

The remainder of this study is organized as follows. Section 2 explains the proposed methodology supported by a case study designed to explore different scenario of implementing autonomous 
technologies for delivery. Section 3 discusses the results after conducting a detailed analysis. Section 4 presents conclusion and future work direction.

\subsection{Methodology}

The performance of transportation systems is of crucial importance for the economic growth. When it comes to the last mile delivery in urban areas, efforts have been made to measure and control traffic movement within highly congested areas using conventional methods. However, with new technologies emerging this research will investigate the effectiveness of incorporating two emerging technologies; autonomous ground vehicles (robots) and autonomous aerial vehicles (drones) as alternative solutions for last mile food delivery. These systems are proposed as a means to providing the same quality of service that cars currently provide without using the road network thus reducing congestion.

\subsubsection{General System Design}

Using the robot and drone technologies three types of delivery systems are designed and evaluated:

1- The autonomous ground vehicle system. This system consists of a fleet of delivery robots (R) that operate on the sidewalk. This system assumes the robots travel at free flow speed with no pedestrian interference or waiting on signalized intersections for permission to cross. The robot system primary benefit is that it can navigate the sidewalks allowing it to bypass traffic easily

2- The autonomous aerial vehicle system. This system will consist of a fleet of drones (D) that operate in a straight distance between two nodes assuming all high-rise buildings are at house level which allows drones to fly straight through. The drone system primary benefit is its ability to deliver at high speeds however there are strict government laws preventing the widespread use of commercial drones. 
3- A hybrid system utilizing both robots and drones. This system contains two phases. Phase one will have robots travel from the depot to the restaurants locations for pick-up and drop the package at the hub. Then phase two comes in, where the package is placed in a drone and sent from the depot to its drop-off destination. The hybrid system will utilize both technologies allowing robots to pick-up orders in highly densely populated areas where the landing of drones maybe difficult due to geographical conditions followed by drones to deliver food to farther locations where landing can be feasible.

\subsubsection{Proposed delivery process for delivery robots and drone systems}

Two different transportation networks are developed and simulated in MATLAB. The robot system network $\mathrm{N}(\mathrm{I}, \mathrm{L})$, consists of I intersections and L links representing roads. As for the

drones system the network $\mathrm{G}(\mathrm{I}, \mathrm{S})$ consists of I intersections and S Links representing the straight distance between two nodes assuming drones can fly without restrictions. Intersections $I=\{\alpha, \beta\}$ can be identified as either Restaurant $(\beta)$ or Home $(\alpha)$.

Both systems consist of two agents: vehicles $(v \in V)$ and food packages $(p \in P)$. In the robot system vehicles are identified as robots $(\mathrm{r} \in \mathrm{R})$. In the drone system the vehicles are identified as drones $(\mathrm{d} \epsilon \mathrm{D})$. Different fleet sizes are tested to determine the most efficient fleet size for three sets of demands (o€O).

A centralized dispatching system (Di) to conduct the matching process is developed. When an order is placed (o), the dispatcher (Di) creates a food request table, which contains package ID, request time, Restaurant node, Home node. To account for meal preparation time the dispatcher adds 12 minutes to all orders requests time before assigning a vehicle to it. From every demand, a set of active orders requests are identified. Active orders (Ao) mean that the customer has placed an order and has not received it yet $\left(\right.$ Ao $=\left\{\right.$ Ao_t $_{-}$Ao_ $\left.\left.\{\mathrm{t}+1\}, \ldots\right\}\right)$. 
After identifying the set of active order requests, every active request needs to be assigned to a vehicle. Therefore, the dispatcher (Di) checks the number of vehicles available every second during the simulation $(\mathrm{t}) . \Delta \mathrm{t}$ is the dispatch update time interval. The matching process policy of assigning a vehicle to an order is based on first-in first-out (FIFO). After determining the vehicle's availability, the dispatcher assigns the available vehicle to the first active order in queue based on a greedy algorithm assigning the vehicle available to the nearest restaurant. For the robot system the dispatcher navigates the robots on the sidewalk network using Dijkstra's shortest path algorithm. Dijkstra's shortest path algorithm operates based on the shortest time depending on the links distance and the speed of the robot. The robots only operate on the sidewalks and cannot navigate highways so certain areas need to be avoided. As for the drone system the dispatcher navigates the drones using a straight-line distance.

Once the delivery has been dropped off to its destination, the vehicle becomes available and can be reassigned to the next order in queue in the food request table. If there are no orders to be assigned, the vehicle remains at the destination node waiting to be assigned when an order comes in queue. The steps used for the robot system are described in detail in Algorithm 1. Furthermore, the methodology and dispatcher processes for the robot system is illustrated in Figures 2 and 3. As for the steps used for the drone system are described in detail in Algorithm 2. Moreover, the methodology and dispatcher processes for the drone system are illustrated in Figures 4 and 5. The simulation keeps running until all the orders that have been placed in an hour have been delivered to the customers. 
Algorithm 1: Pseudo-code for Robots Food Delivery System

1. $\mathrm{t}=1$;

2. Customer places order through the food delivery app;

3. Dispatcher (Di) identifies set of active requests Ao $=\left\{\right.$ Ao_t, Ao_ $\left._{-}\{\mathrm{t}+1\}, \ldots\right\}$;

4. while active requests (Ao) exists do

5. Dispatcher (Di) creates a food request table based on orders placed in a chronological order;

6. $\quad$ Dispatcher (Di) starts searching for available robots (r_av) in the network;

7. If available robots (r_av) are found

8. $\quad$ Check all ( $\mathrm{r} \_a v$ ) distances from the first order in queue;

9. $\quad$ Dispatcher (Di) assigns the first request lined up in queue to the nearest available vehicle to the restaurant for pick-up;

10. The robot travels from its location to the assigned restaurant for pick-up using Dijkstra's shortest path algorithm;

11. Dispatcher (Di) updates list of vehicles available and orders in queue;

12. $\quad$ else

13. Dispatcher (Di) waits until a robot in use becomes available to assign the food package;

$14 . \quad$ end

15. $\mathrm{t}=\mathrm{t}+1$;

16. end

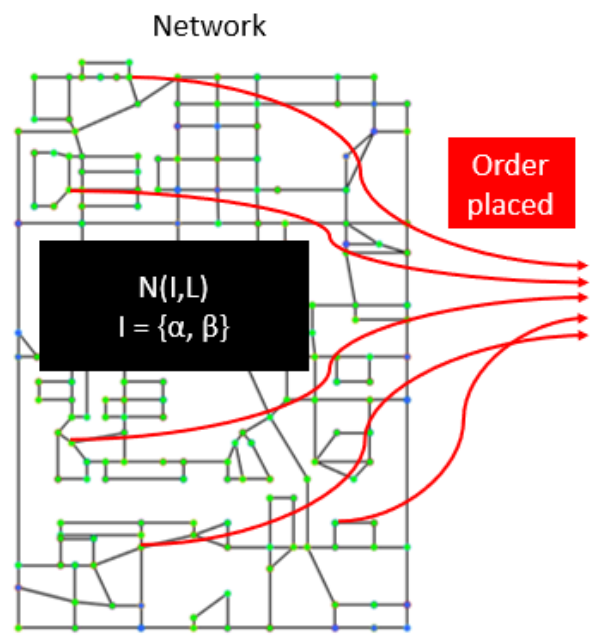

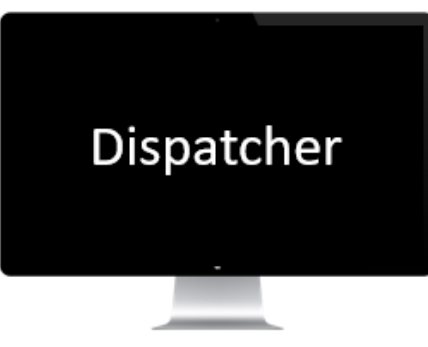

Matching process

Navigation process
Food Request Table

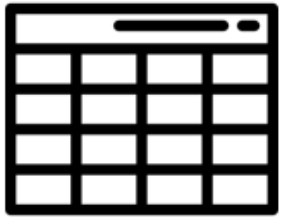

Package ID

Request time $+12 \mathrm{~min}$

Restaurant node (O)

Home node (D)

FIGURE 2 : Methodology for robot system 


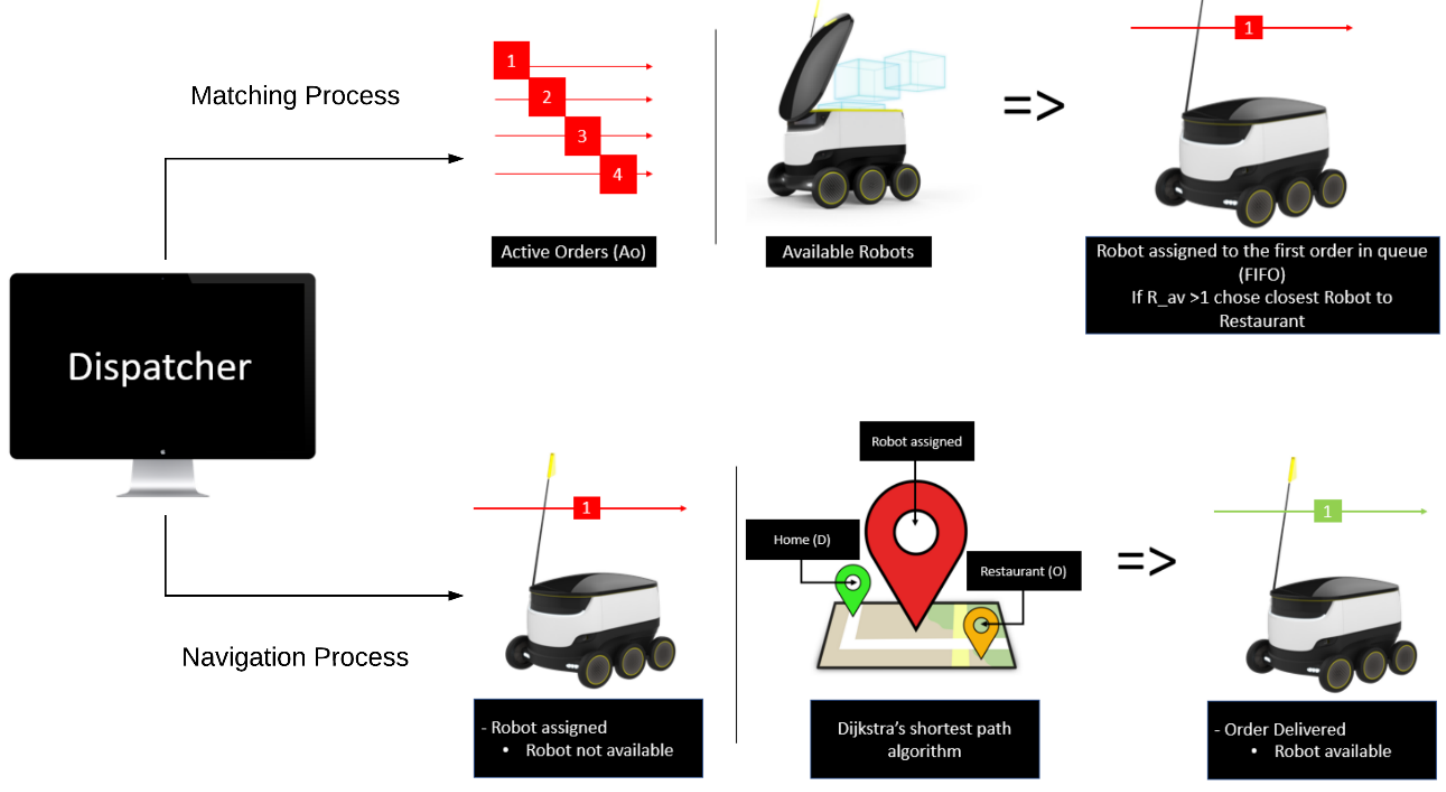

FIGURE 3 : Dispatcher processes for robot system

Algorithm 2: Pseudo-code for Drones Food Delivery System

1. $\mathrm{t}=1$;

2. Customer places order through the food delivery app;

3. Dispatcher (Di) identifies set of active requests Ao $=\left\{\right.$ Ao_t $\left., A o \_\{t+1\}, \ldots\right\}$;

4. while active requests (Ao) exist do

5. Dispatcher (Di) creates a food request table based on orders placed in a chronological order;

6. $\quad$ Dispatcher (Di) starts searching for available drones in the network (d_av);

7. If available drones (d_av) exist

8. $\quad$ Check all (d_av) distances from the first order in queue;

9. Dispatcher (Di) assigns the first request lined up in queue to the nearest available vehicle to the restaurant for pick-up;

10. $\quad$ The drone travels from its location to the assigned restaurant for pick-up;

11. Dispatcher (Di) updates list of drones available(d_av) and orders in queue;

12. else

13. Dispatcher (Di) waits until a drone in use becomes available (d_av) to assign the food Package to;

14. end

15. $\mathrm{t}=\mathrm{t}+1$;

16. end 

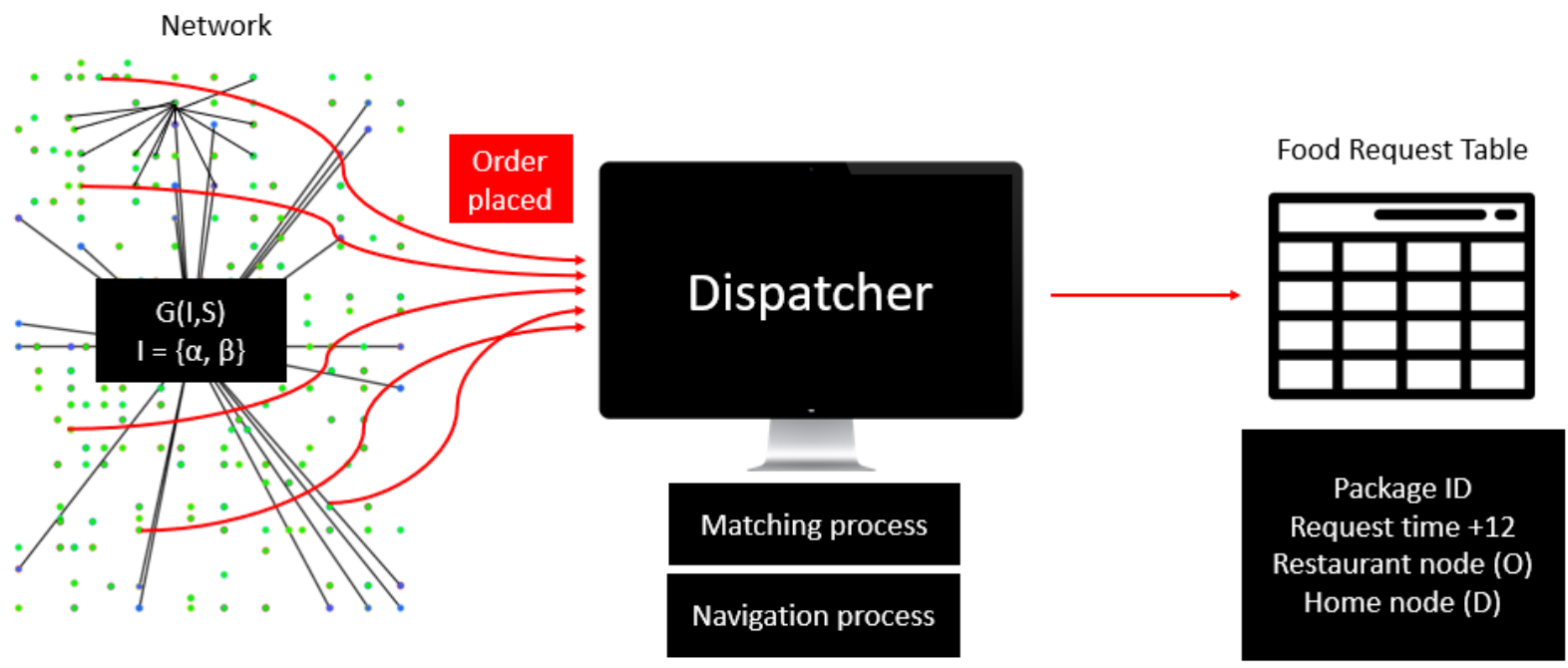

FIGURE 4 : Methodology for drone system

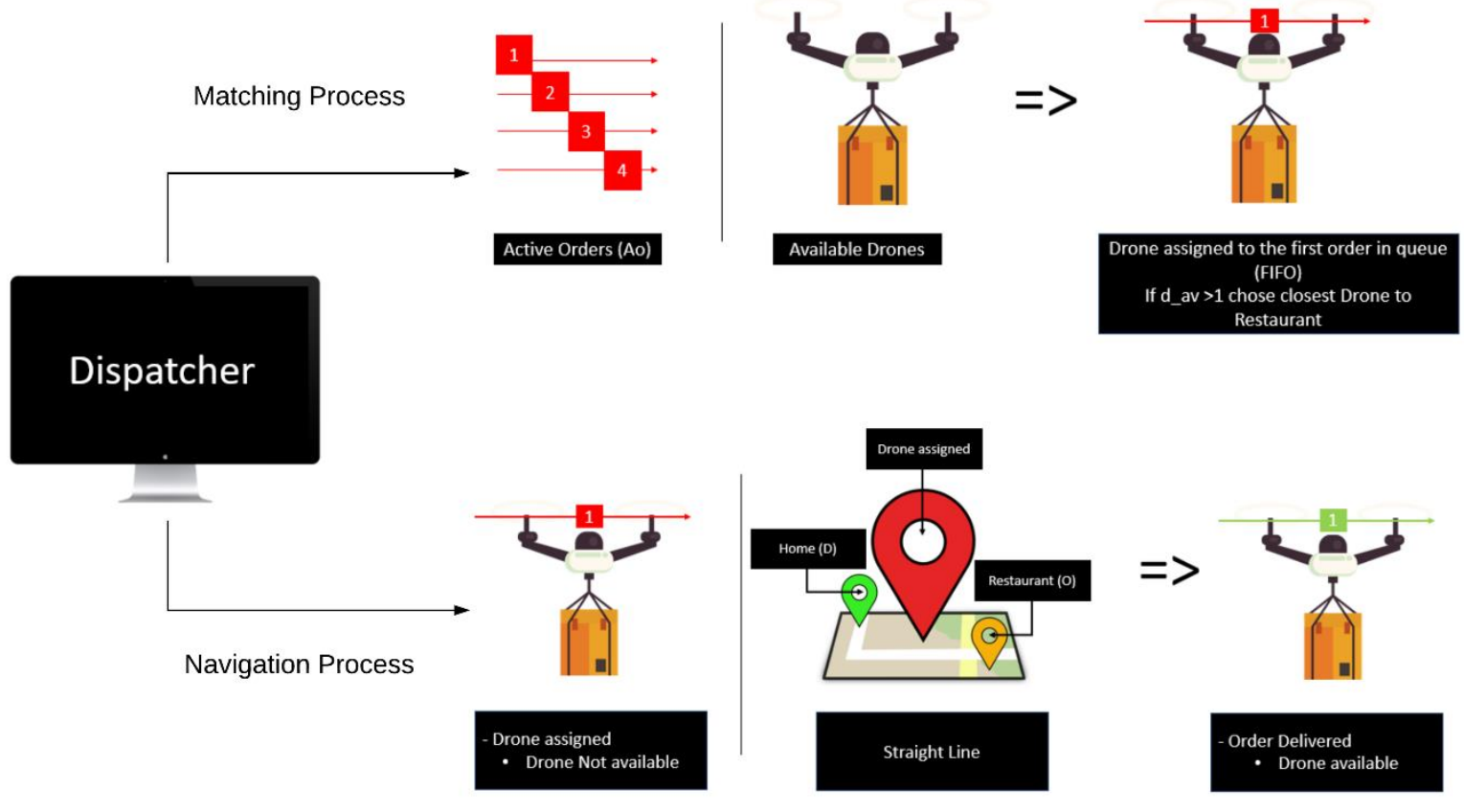

FIGURE 5 : Dispatcher processes for drone system 


\subsubsection{Proposed delivery process for hybrid delivery system}

The major significant difference of this system is that it utilizes both robots and drones together operating from a hub/depot. There are two main benefits associated with such a hybrid system: 1) one or more hubs can be strategically placed across the network where food from different restaurants can be consolidated together through a hub and be sent directly to their destinations. Such a system is called hub-and-spoke network and has been used by airline companies and in recently years by logistic companies to reduce transportation costs, improve cycle times and reduce inventory and more importantly serve more destinations for a given volume of output [39]. Having one or more hubs also allows but aerial and ground vehicles to recharge and be maintained while not in use, making this system a fully integrated system. Jung and Jaykrishnan [40] used the concept of hub-and-spoke network to increase the coverage point of electric shared taxis. 2) using both technologies at once allows the system to reap the benefits of these technologies since they possess different characteristics from their speeds to their navigation and trajectory systems. Both networks are used to operate this system and Intersections $I=\{\alpha, \beta, \mu\}$ can be identified as either Restaurant $(\beta)$, Home $(\alpha)$ or Depot $(\mu)$.

This is system is divided into two phases; phase one uses robots to go from the depot $(\mu)$ to pick up the food package at the restaurant $(\beta)$ and drops it off back at the depot $(\mu)$. The first phase was assigned to robots due to the robot characteristics of having lower speeds and operating on the sidewalks allowing the restaurant sufficient time to prepare the meal and therefore the dispatcher is not required to add 12 min to account for food preparation time. Phase two uses drones to carry the food package from the $\operatorname{depot}(\mu)$ to the home $(\alpha)$ and heads back to the depot empty $(\mu)$. Phase two was chosen as drones because of their higher speeds and the fact that they need a designated landing area to place the food. For this system one centralized dispatcher is created. However, two 
food request tables are generated from it; robot food request table $(\Upsilon)$ and drone food request table $(\tau)$

Once a food order (o) is placed by a costumer, the order (o) is placed in the robot food request table $(\Upsilon)$. An available robot $\left(r_{-}\right.$av) residing in the $\operatorname{depot}(\mu)$ is assigned to pick-up the first package in queue from the restaurant $(\beta)$ and bring it back to the depot $(\mu)$. After dropping off the food package (p) at the depot the order is assigned to the drone food request table $(\tau)$ with a time stamp equivalent to the robot drop-off time of the package. When an available drone is located in the $\operatorname{depot}(\mu)$, it gets reassigned to the first package in queue and delivers the (p) from the depot $(\mu)$ to the home $(\alpha)$ thus completing the delivery of the order (o). The details for the hybrid system are described in Algorithm 3 and the methodology is illustrated in Figure 6 and 7. 
Algorithm 3: Pseudo-code for Hybrid Food Delivery System

1. $\mathrm{t}=1$;

2. Customer places order through the food delivery app;

3. Dispatcher (Di) identifies set of active requests Ao $=\{$ Ao_t, Ao_ $\{\mathrm{t}+1\}, \ldots\}$;

4. while active requests (Ao) exist do

5. $\quad$ Dispatcher $(\mathrm{Di})$ creates a robot food request table $(\Upsilon)$ based on orders placed in a chronological order;

6. Dispatcher (Di) creates a drone food request table $(\tau)$;

Dispatcher (Di) assigns available robot ( $\left.r \_a v\right)$ to the first active request lined up in queue (Ao_t $)$ in $(\Upsilon)$;

(r) travels from depot $(\mu)$ to the assigned restaurant $(\beta)$ for pick-up;

(r) drops of $($ Ao_t $)$ in depot $(\mu)$;

Dispatcher (Di) updates list of robots available $\left(\mathrm{r}_{-} \mathrm{av}\right)$ at depot $(\mu)$;

Dispatcher (Di) updates the set of orders (Ao_t $)$ in $(\Upsilon)$;

when (Ao_t) reaches depot $(\mu)$ do;

Dispatcher places (Ao_t) in queue in $(\tau)$;

If drone available (d_av) in depot $(\mu)$;

Dispatcher (Di) assigns available drone(d_av) to the first active request lined up in queue (Ao_t) in $(\tau)$;

(d) travels from its location to the assigned home $(\alpha)$ for drop-off;

(d) drops-off (Ao_t) at home $(\alpha)$;

(d) returns to depot $(\mu)$;

Dispatcher (Di) updates list of drones available(d_av) at depot $(\mu)$;

Dispatcher (Di) updates the set of orders (Ao_t $)$ in $(\tau)$; else

| Dispatcher (Di) waits until a drone becomes available in the depot $(\mu)$; end

else

Dispatcher (Di) waits until a robot becomes available in depot $(\mu)$ to assign it to $($ Ao_t $)$;

end

$\mathrm{t}=\mathrm{t}+1$

end 

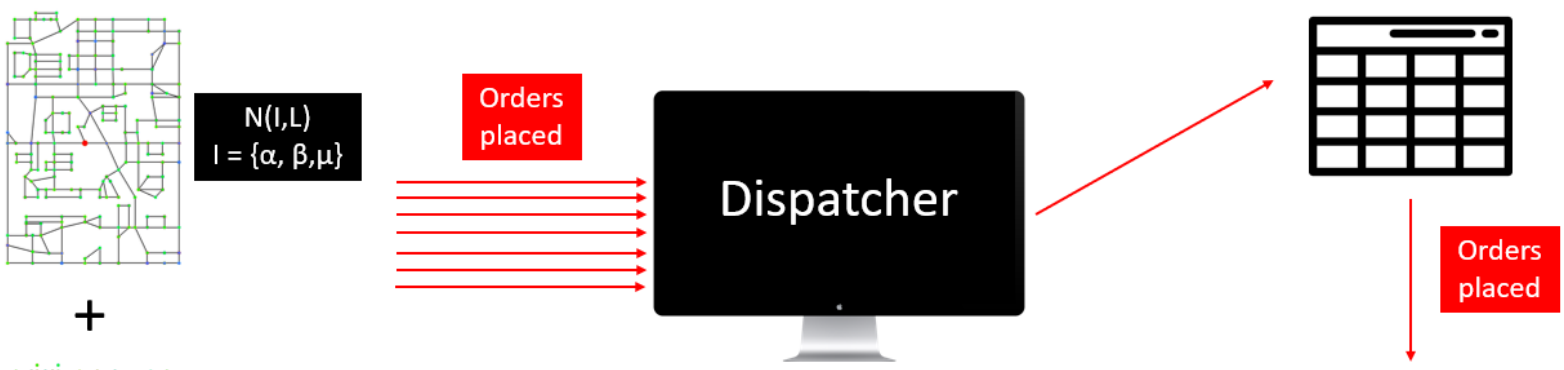

Matching process

Drone Food Request Table

\section{$\mathrm{G}(\mathrm{I}, \mathrm{S})$}

$\mathrm{I}=\{\alpha, \beta, \mu\}$

Navigation process

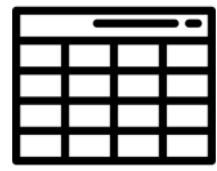

FIGURE 6 : Methodology for hybrid system 1.0

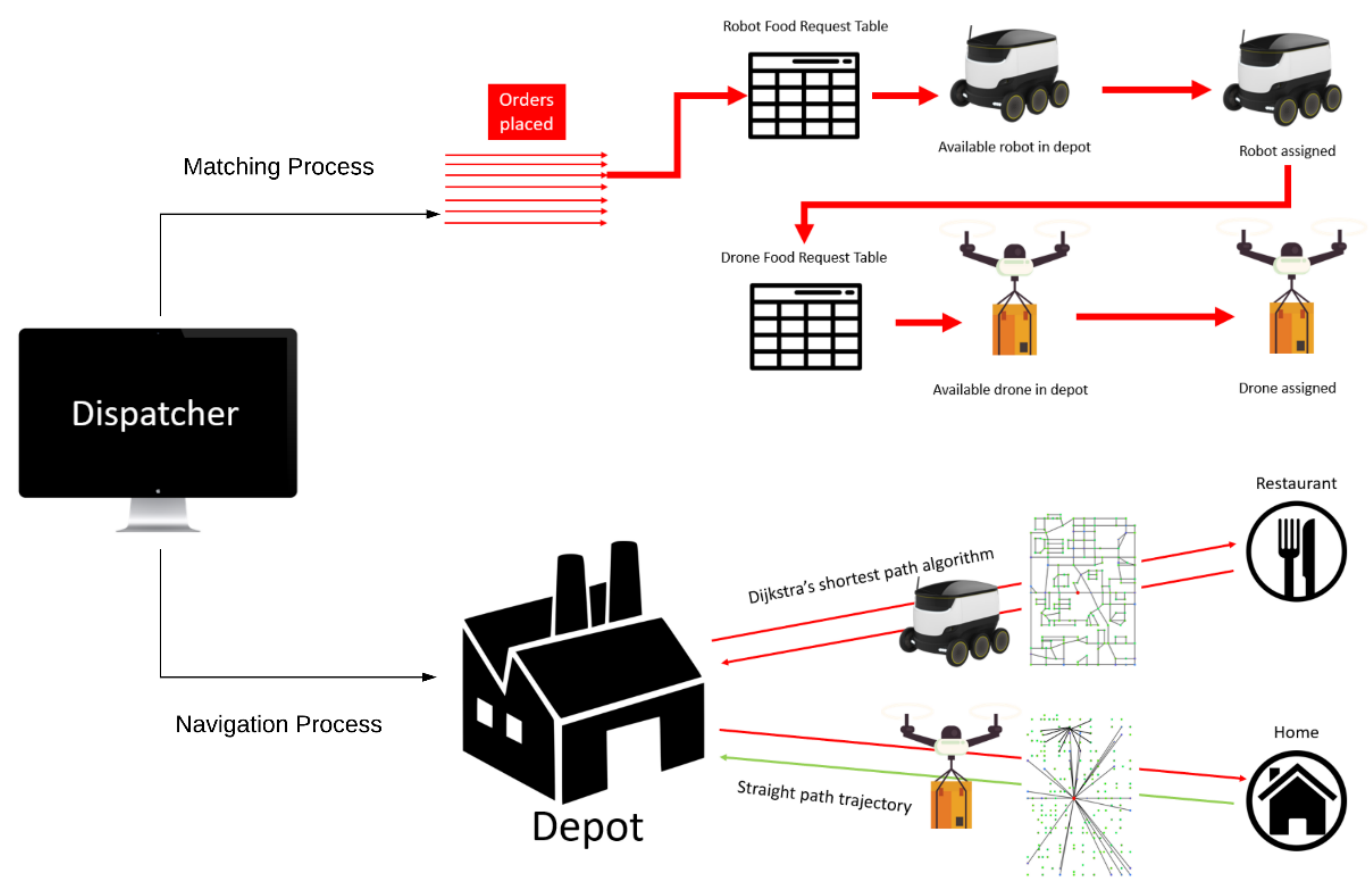

FIGURE 7 : Dispatcher processes for hybrid system 1.0 


\subsubsection{Performance criteria for all delivery systems}

To evaluate the performance of the three proposed systems, a Level of Service (LOS) scale is developed based on the customer average waiting time (w). Based on UberEATS business model identifying wait times for each restaurant from (15 minutes to 50 minutes) depending on the type of cuisine the restaurant services along with reference to the vehicle traffic Level of Service Categories and the criteria used in dividing those categories, a generic performance criterion in Table 1 was developed for all three systems. LOS should be evaluated for the overall delivery system and for each order separately to determine the optimal solution.

For the system overall LOS reached category implies the following:

LOS A: Over reliable system

LOS B: Reasonably reliable system

LOS C: Stable system

LOS D: Approaching unreliable state system

LOS F: Completely unreliable system

TABLE 1 Level of Service Criteria

\begin{tabular}{|c|c|}
\hline LOS & Average Wait Time (min) \\
\hline A & $1-20$ \\
\hline B & $21-30$ \\
\hline C & $31-40$ \\
\hline D & $41-50$ \\
\hline F & 51 or more \\
\hline
\end{tabular}




\subsection{Case Study}

For our case study the City of Mississauga in Ontario was chosen due to its highly dense populated urban area, being ranked the $6^{\text {th }}$ most populated among cities and towns across Canada [41]. The road network was developed in MATLAB with an approximate area of $\left(5.80 \mathrm{~km}^{2}\right)$ capturing the area as shown in Figure 8 with a total of 199 intersections and 286 streets links for robots to navigate the sidewalk. The red dot represents the hub location for the hybrid system.

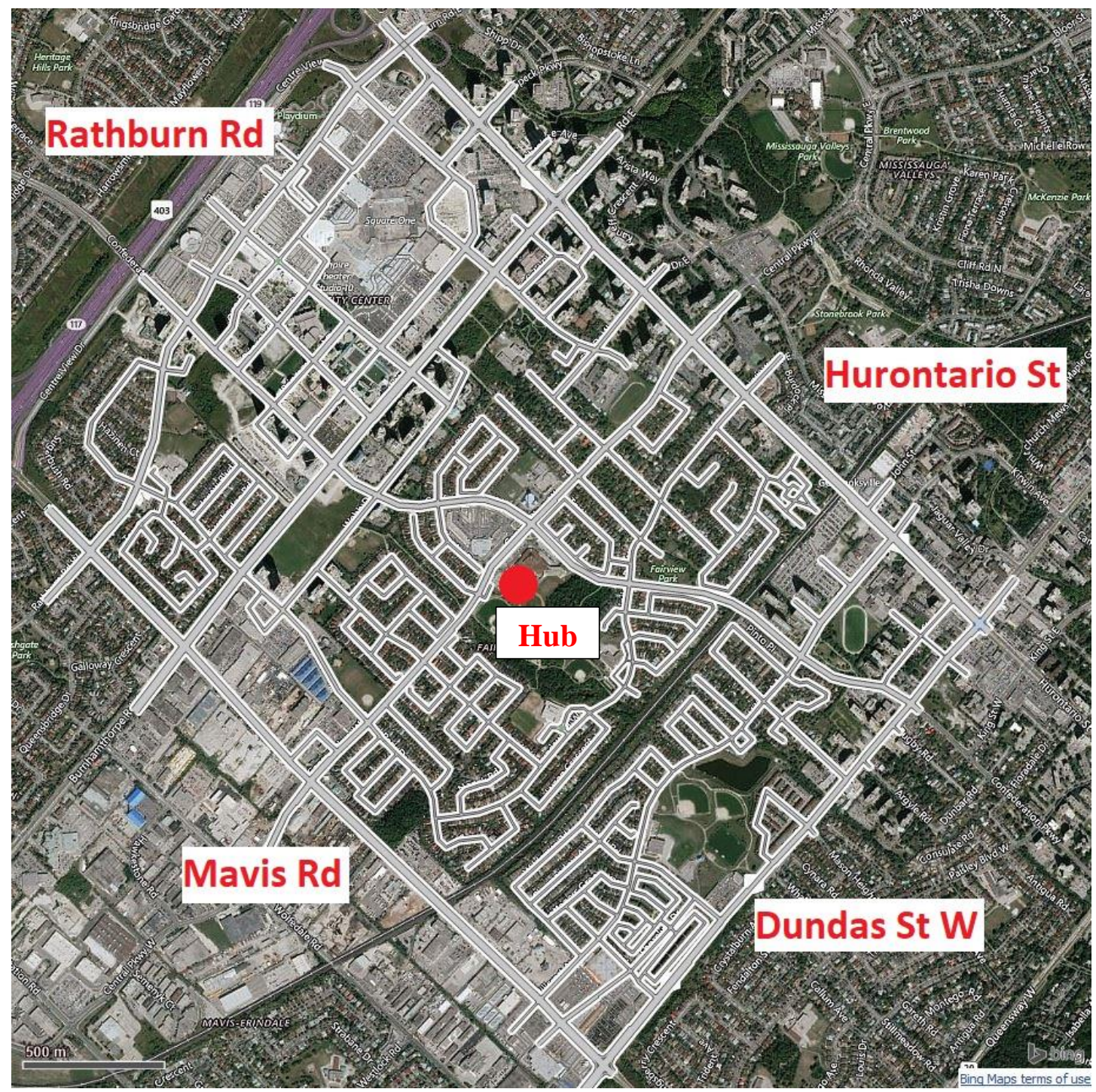

FIGURE 8 Mississauga network. 
The area chosen is considered Mississauga city's downtown core consisting of both commercial and residential areas. However, the different land uses are not distributed uniformly. Downtown core consists of multiple small to medium commercial compounds as well as one major commercial compound known as Square One shopping Centre. Various restaurants exist within Square One shopping Centre and its surroundings. Furthermore, the area chosen has a high population density with multiple high-rise buildings due to its strategic location in downtown Mississauga.

In the simulation model nodes are identified as one of the following three: Restaurant, House, Depot. UberEATS website is used to identify all the restaurants within the boundaries or surrounding the edges. The nearest node to the actual restaurant locations are as signed as a restaurant node (origin nodes) and all the other nodes, which are not restaurants, were identified as house nodes (destination nodes). Furthermore, the location of depot is chosen based on the central location in the test network, where the number of nodes above the depot are approximately the same as below it, as well as on both sides from left and right. In this study due to small size of the studied network only one hub/depot is considered however the methodology is easily extendable to multiple hubs. In addition, the node assigned as depot is located near a park location so the design would be practical and there is sufficient space for an actual depot to be built. The study period for the simulation is during the evening peak period from 5:00 p.m. $-6: 00$ p.m. The demand used in this study is time dependent exogenous demand origin-destination matrices. From a set of restaurant nodes and house nodes, orders were created by matching a restaurant with a corresponding home node through uniform distribution creating an OD matrix. Time stamps assigned were uniformly distributed to each order between 5:00 p.m. - 6:00 p.m. During the study period, three demand scenarios were tested having the original demand set as 340 orders and 
increasing the demand by $10 \% \& 20 \%$ growth rates. Moreover, different fleet sizes are tested for each delivery system. In total 18 scenarios are investigated as shown in Table 2 to compare the performance of the three proposed last mile delivery systems in terms of wait time and optimal starting fleet size required. We used larger fleet sizes for robot system than drones fleet sizes due to two primary factors:

1- The robots have a speed of approximately $4.5 \mathrm{~m} / \mathrm{s}$ while the drones have a significantly higher speed of $40 \mathrm{~km} / \mathrm{hr}$. The speed of robots was determined based on the speed of a Starships Company food delivery robot as for the drones the speed was set as half the maximum speed for an Amazon air prime delivery drone accounting for time to take off, landing time as well as the extra weight it may carry.

2- Robots have to follow a designated road network while drones can fly straight distances

In the hybrid system we combine both robots and drone system and activate a depot location to develop a fully integrated autonomous delivery system that optimizes the benefits of using the two previous systems.

TABLE 2 List of Simulation Scenarios

\begin{tabular}{|c|c|c|c|}
\hline Scenario number & \multicolumn{1}{|c|}{ System } & Fleet Size & Demand \\
\hline $\mathbf{1}$ & Robot System & 25 robots & Original, 10\%, 20\% \\
\hline $\mathbf{2}$ & Robot System & 50 robots & Original, 10\%, 20\% \\
\hline $\mathbf{3}$ & Robot System & 75 robots & Original, 10\%, 20\% \\
\hline $\mathbf{4}$ & Robot System & 100 robots & Original, 10\%, 20\% \\
\hline
\end{tabular}




\begin{tabular}{|c|c|c|c|}
\hline 5 & Robot System & 125 robots & Original, $10 \%, 20 \%$ \\
\hline 6 & Robot System & 150 robots & Original, $10 \%, 20 \%$ \\
\hline 7 & Drones System & 5 drones & Original, $10 \%, 20 \%$ \\
\hline 8 & Drones System & 10 drones & Original, $10 \%, 20 \%$ \\
\hline 9 & Drones System & 15 drones & Original, $10 \%, 20 \%$ \\
\hline 10 & Drones System & 20 drones & Original, $10 \%, 20 \%$ \\
\hline 11 & Drones System & 25 drones & Original, $10 \%, 20 \%$ \\
\hline 12 & Drones System & 30 drones & Original, $10 \%, 20 \%$ \\
\hline 13 & Hybrid System & 25 robots -10 drones & $20 \%$ increase \\
\hline 14 & Hybrid System & 25 robots -15 drones & $20 \%$ increase \\
\hline 15 & Hybrid System & 25 robots -20 drones & $20 \%$ increase \\
\hline 16 & Hybrid System & 30 robots -10 drones & $20 \%$ increase \\
\hline 17 & Hybrid System & 30 robots -15 drones & $20 \%$ increase \\
\hline 18 & Hybrid System & 30 robots -20 drones & $20 \%$ increase \\
\hline
\end{tabular}




\subsubsection{Food Delivery Systems Operating Policy Simulation}

The simulation time step is 1 second as well as the dispatcher update time interval. The simulation ends when all the food packages are dropped off at their destination and the food request table is cleared out.

For the robot and drone system, an example of a vehicle itinerary from the robot system with a fleet size of 75 robots operating at $20 \%$ increased demand set in Mississauga case study is presented in Table 3. Note that vehicle gets assigned after adding food preparation time.

Where: o_ID: order ID; R_t: Order Request time (seconds) ; FP_t: Food Preparation Time (seconds) ; $\beta$ : Origin node (Restaurant); $\alpha$ : Destination node (Home); P_t: Pick-up time from Restaurant (seconds); D_t: Drop-off time at Home (seconds); W_t: Customer wait time (seconds).

TABLE 3 Sample of Robot (1) Itinerary

\begin{tabular}{|c|c|c|c|c|c|c|c|}
\hline $\mathbf{0} \_$ID & R_t & FP_t & $\boldsymbol{\beta}$ & $\boldsymbol{\alpha}$ & P_t & D_t & W_t \\
\hline $\mathbf{1}$ & 2 & 720 & 4 & 90 & 1204 & 1633 & 1631 \\
\hline $\mathbf{9 1}$ & 830 & 720 & 126 & 56 & 2063 & 2319 & 1489 \\
\hline $\mathbf{1 5 0}$ & 1263 & 720 & 148 & 113 & 2734 & 3311 & 2048 \\
\hline $\mathbf{2 4 1}$ & 2111 & 720 & 10 & 190 & 3923 & 4776 & 2665 \\
\hline $\mathbf{3 7 4}$ & 3293 & 720 & 167 & 131 & 5022 & 5377 & 2084 \\
\hline
\end{tabular}


An example of robot 1 path nodes that corresponds with order ID 374 is as follows:

Vehicle (1).Pathnode $=$

$[190,189,154,155,160,163,166,167,120,121,124,125,126,127,133,132,131]$

For the robot routing system, it follows the Dijkstra's Shortest Path algorithm, and once the robot drops-off the order at the house node location it remains there until it gets reassigned to pick-up the next order. For the drones routing system, it follows a direct straight link connecting the nodes.

For the hybrid system, an example of an order going through the system with a robot fleet size of 25 and a drones fleet size of 15 operating at $20 \%$ increased demand set in Mississauga case study is presented in Table 4.

Where: o_ID: order ID; R_t: Order Request time (seconds); $\beta$ : Origin node (Restaurant); $\alpha$ : Destination node (Home); rP_t: Robot Pick-up time from Restaurant (seconds) ; rD_t: Robot Dropoff time at depot (seconds) ; dP_t: Drone Pick-up time from depot (seconds) ; dD_t: Drone Dropoff time at home (seconds) W_t: Customer wait time (seconds)

TABLE 4 Sample of Order (4) Itinerary

\begin{tabular}{|l|l|l|l|l|l|l|l|l|}
\hline $\mathbf{0} \_$ID & R_t & $\boldsymbol{\beta}$ & $\boldsymbol{\alpha}$ & rP_t & rD_t & dP_t & dD_t & W_t \\
\hline 114 & 1018 & 10 & 70 & 1532 & 1768 & 1768 & 1962 & 944 \\
\hline
\end{tabular}

For the routing system used, since we are using both autonomous technologies; robots and drones, the robots phase uses the road network and follows the Dijkstra's Shortest Path algorithm, while 
the drones follow the straight trajectory between any two nodes therefore not needing to follow any routing algorithm

As can be seen from Table 4, robot pickup time was after 514 seconds from when the order was placed denoting that there were no available robots at the time the request was made. The order gets placed in queue for an available robot to return to the depot. Moving on to phase two, since there was an available drone at the depot the order got picked-up immediately after being droppedoff at the depot and was dropped-off to the client totaling up the costumers wait time to 944 seconds.

\subsection{Results \& analysis}

Figure (9) and (10) present the average wait time for robot and drone systems according to different demand scenarios. The results show that for the robot and drone system, when increasing the fleet sizes it reduces the average wait time for customers which is an apparent conclusion because when we have more vehicles in the system the better the performance of the system will be however, after increasing the fleet size the average waiting time plateaus. For the robot system the wait time starts to level around 100 robots as for the drone system it starts to plateau after 15 drones reaching a level state from 20 drones onwards for our three sets of demand. We can determine from Figure 9 and 10 that the optimal starting fleet size for our demand scenarios for robots and drones are; 75 robots and 10 drones with an average wait time for the maximum demand being 32.4 minutes and 28.8 minutes respectively.

However, there is an approximate $87 \%$ difference in optimal starting fleet sizes between the robot system and the drone system, which results in an $11 \%$ difference in the average wait time due to the difference in speed between robots and drones. Therefore, the hybrid solution was introduced to maximize the benefits of both autonomous technologies an activate a depot placed at a strategic 
location to accommodate for the uneven distribution of commercial and residential areas within our study area.

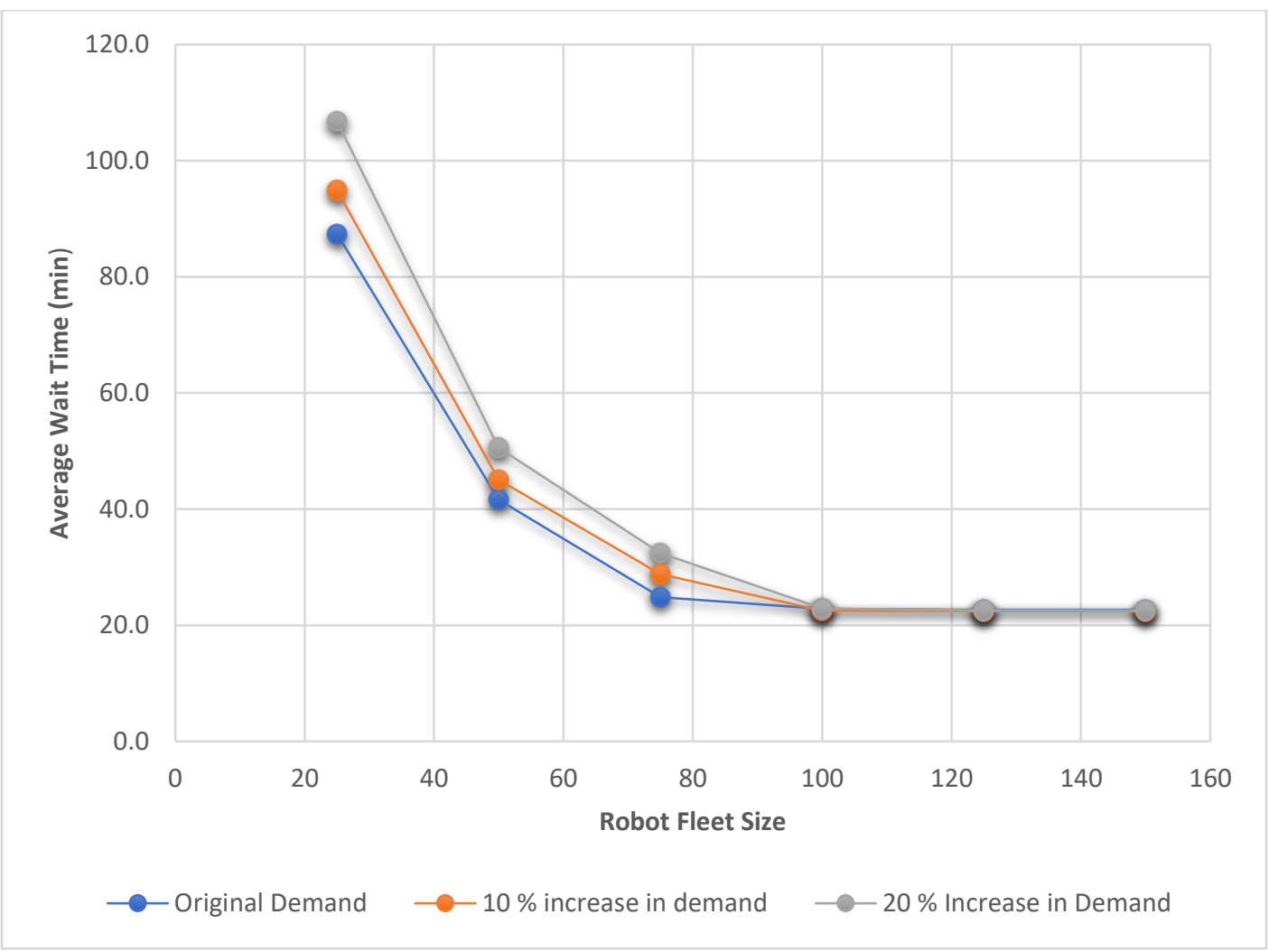

FIGURE 9: Average customer wait time for robots system. 


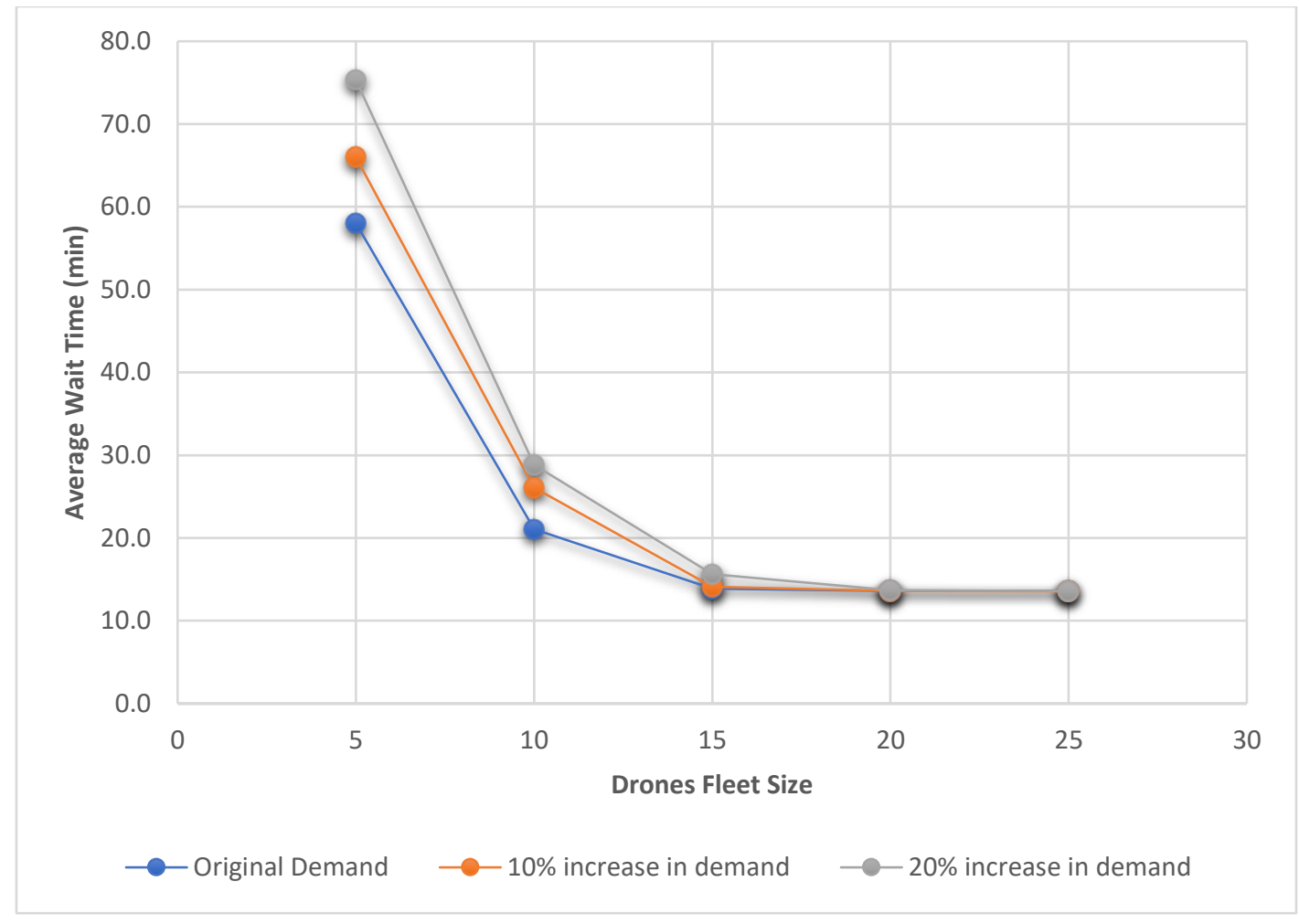

FIGURE 10 : Average customer wait time for drones system.

As shown in Figure 11, for the hybrid delivery system, six different scenarios are analyzed. It can be seen that wait time fluctuated between the six scenarios however, one thing stood out was the number of drones played a significant role in increasing or decreasing the average wait time due to the drone's capability of delivering the food at significantly higher speeds. Therefore, adding 5 drones with keeping 25 robots reduced the average delivery time by approximately $50 \%$. The optimal starting fleet size was having 25 robots and 15 drones. While this hybrid system has an overall LOS C, stable system, based on the average wait time, it is not sufficient to look at only the LOS of the system. The LOS of each order should be assessed because from the average wait time it appeared that 25 robots and 10 drones provides a stable system but in fact there was a high 
percent of orders that fell in the LOS F category as displayed in Figure 12, meaning some orders had to wait a long time in queue until a drone became available.

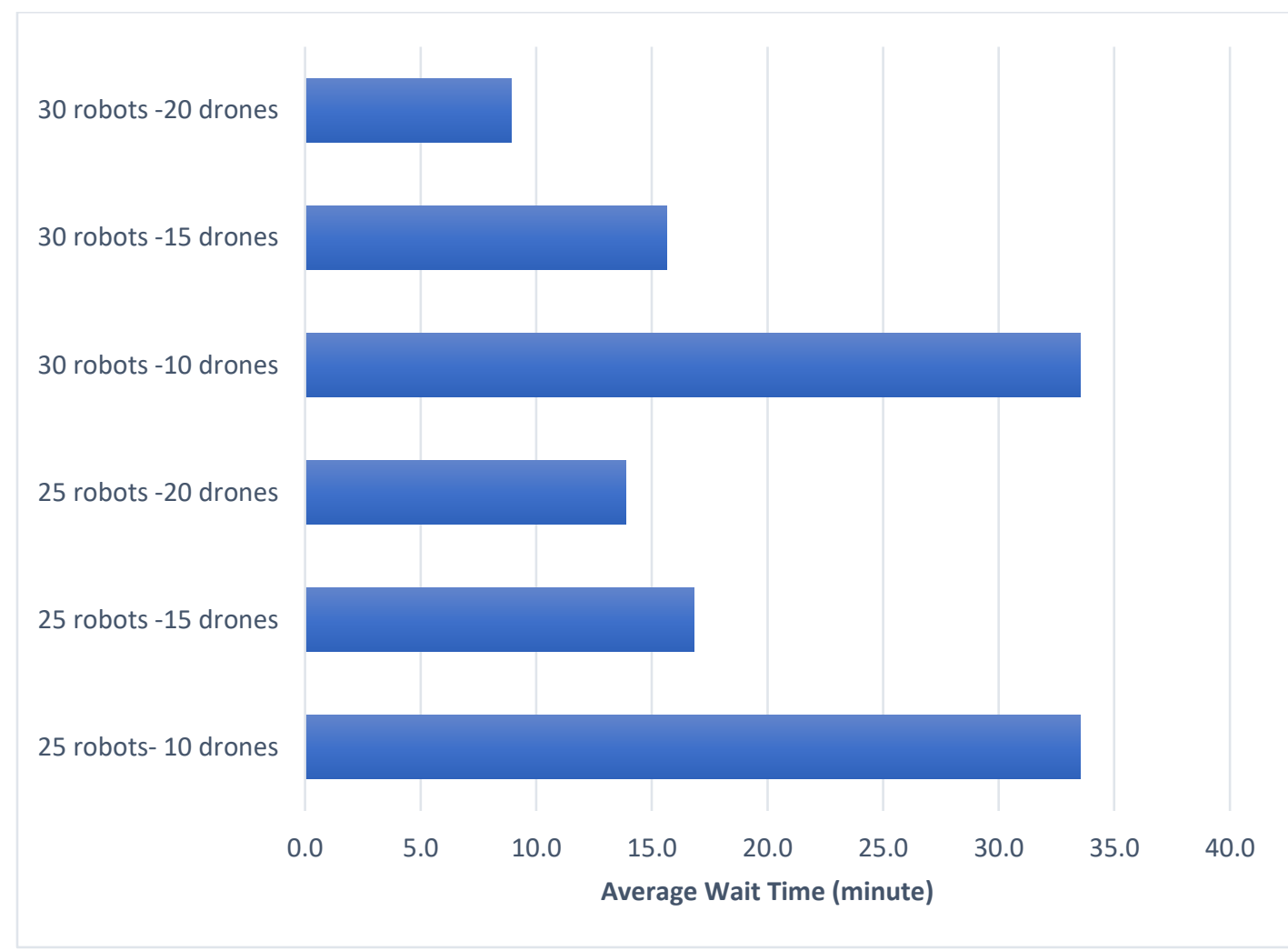

FIGURE 11 : Average wait time for hybrid system 


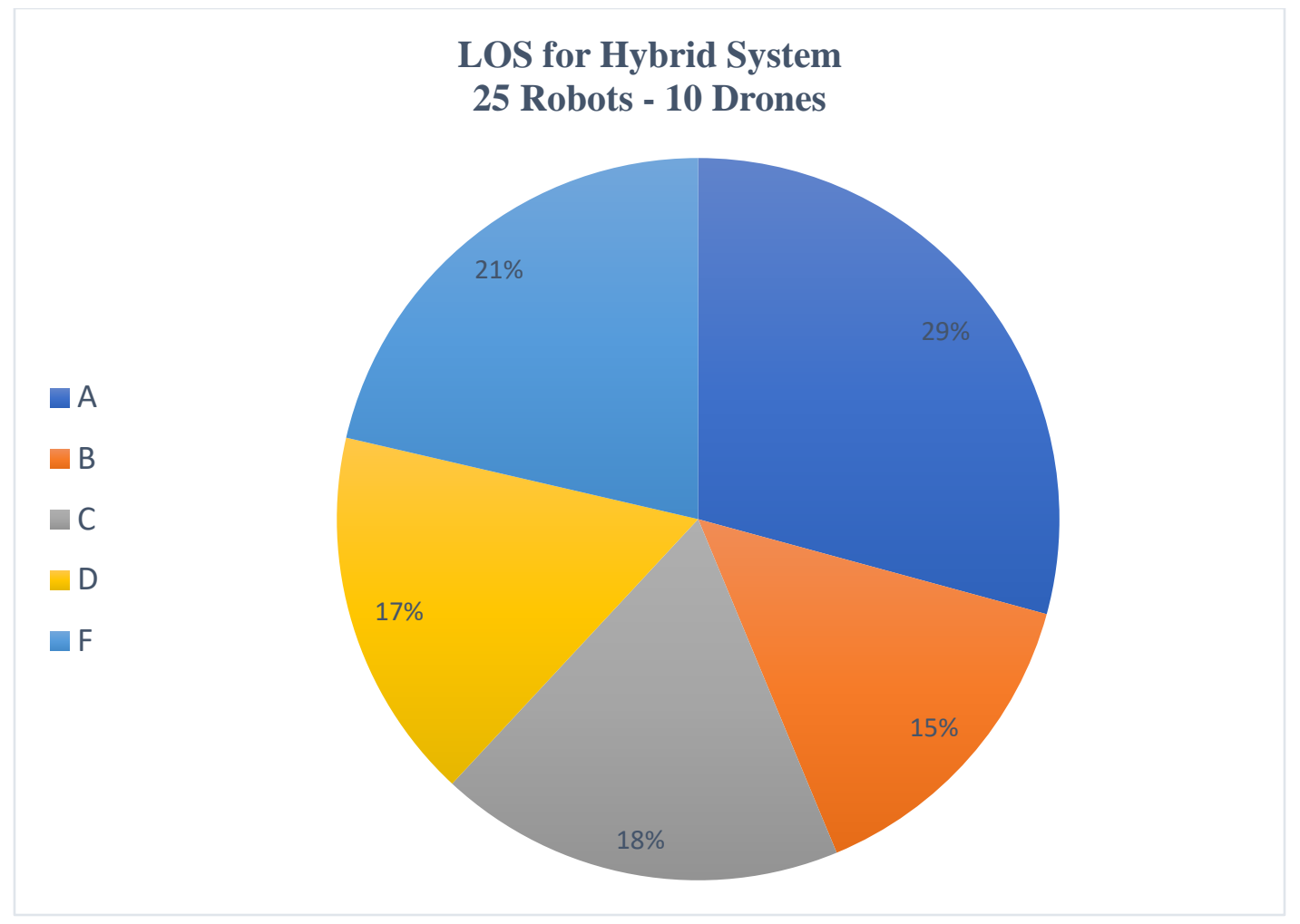

FIGURE 12 : Level of service for scenario 25 robots - 10 drones in the hybrid system

To compare all three system, a box plot is developed as demonstrated in to Figure 13 with the optimal starting fleet sizes determined previously per system. As demonstrated the maximum wait time for the optimal starting robot fleet size is 50 minutes, according to UberEATS website delivery time is usually set between 45-50 minutes per order. However, when applying the drone system, the maximum delivery times reduces by approximately $12 \%$. Furthermore, when applying the hybrid system, it reduces the maximum wait time from the robot system by $43 \%$ and from the drone system by $34 \%$ making the hybrid system the most efficient of all three systems. However, when analyzing the minimum wait time for the hybrid system its 2.5 minutes, which may not be sufficient to some restaurants that are located closer to the depot to prepare the food therefore food preparation time should be allocated to the restaurants that are located near the hub before 
assigning a robot for pick-up. When analyzing the median waiting time, it is observed that for robots and drones median waiting times are less than $10 \%$ apart compared to each other. However, when comparing the median waiting time for the robot system with the hybrid system, the waiting time is reduced by $46 \%$ under hybrid scenario. For the robot and drone systems $50 \%$ of the orders are delivered above (30-33) minutes whereas for the hybrid system 50\% of the orders are delivered more than 17 minutes and up to 29 minutes.

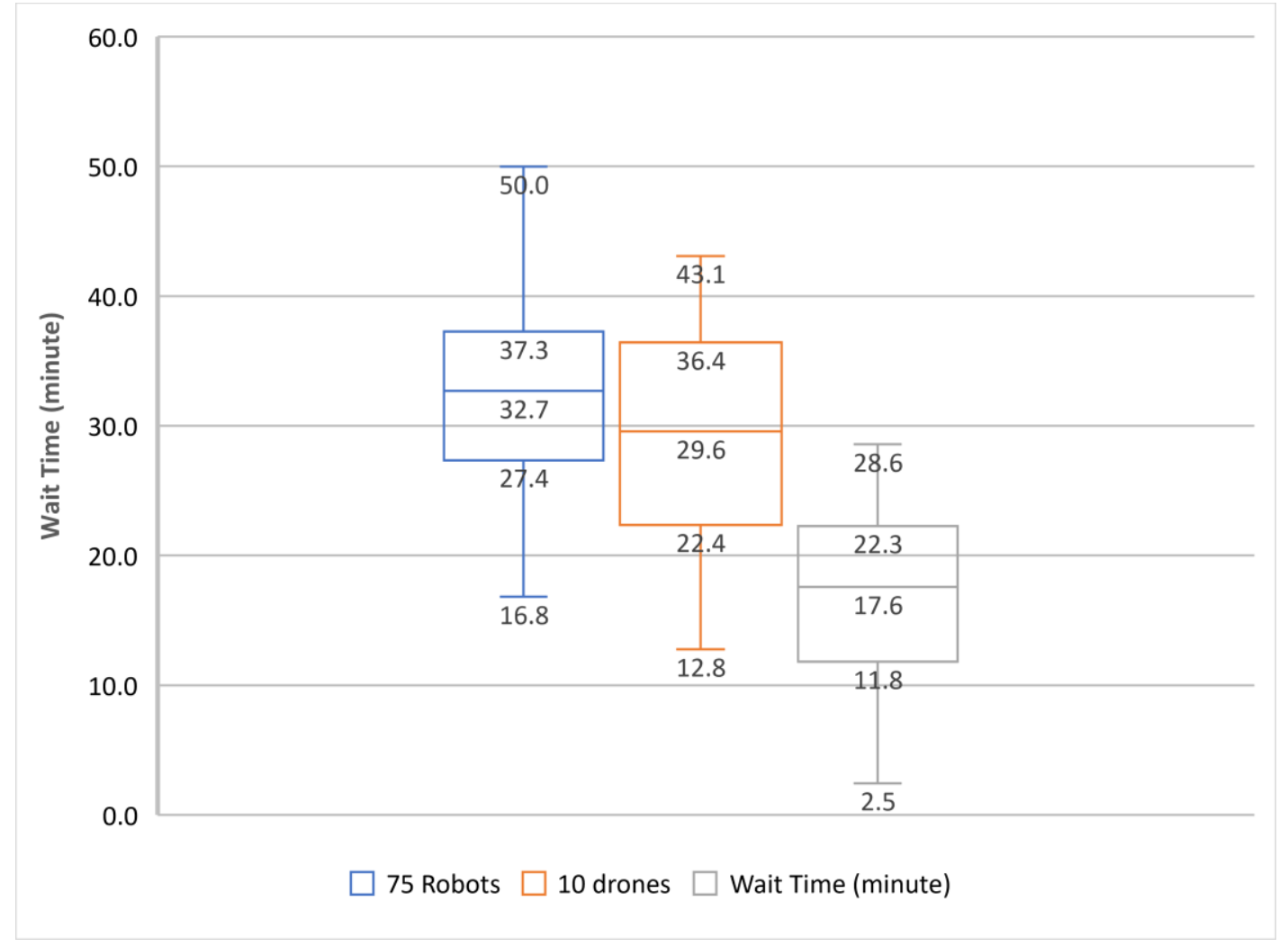

FIGURE 13 : Average wait time for optimal starting fleet sizes per system 


\subsection{Conclusion}

In this study three innovative last mile food delivery systems are proposed employing aerial and ground autonomous vehicles in order to mitigate traffic congestion caused by new and upcoming on-demand food delivery apps. Three systems proposed are: robot-based delivery system, dronebased delivery system and a hybrid system integrating robots and drones.

To test the efficiency of the three proposed systems, they were applied to city of Mississauga in an in-house developed simulation platform during evening peak period. In total 18 scenarios were tested where demand and fleet size were systematically increased. Average wait time and optimal starting fleet size were used as performance measure. The results obtained from simulation showed that for the robot system, a large fleet size is needed to accommodate the applied demand, since the robots operate on the sidewalks and on a lower speed. As for the drones, while a significantly lower fleet size ( $86 \%$ less than robots) was used, there are strict regulations on operating drones for commercial use. Furthermore, with the existence of high-rise buildings processionary measures need to be made for landing as well as adjustments to the drone's trajectory.

For the hybrid system, both technologies were incorporated in the system and a strategic depot location was identified making a hub-and-spoke network. Robots were chosen for phase one of the delivery due to their slower speed, giving time to the restaurant for food preparation, followed by phase two which used drones to drop-off the food The hybrid system was found to be the most efficient of the three systems since it accommodates for the food preparation phase as well as has a depot location that can be used for storage and charging purposes as well as distribution center.

The results showed that at maximum operating demand the hybrid system with optimal starting fleet size had the least average delivery time being $48 \%$ less than the optimal starting fleet size of 
robots and $42 \%$ less than the optimal starting fleet size of drones, with the least variance between orders delivery times.

For future research, a number of actions can be taken to further develop these systems. In current design, customers are served based on (FIFO). However, for the future policies an algorithm can be developed to optimize the assignment of vehicles to orders. Furthermore, to develop a fully integrated system, autonomous vehicles can be added to these systems and can account for delivery sizes that cannot be carried by robots or drones (e.g. catering services). Moreover, the current three systems proposed carry one order at a time. However, for future purposes these systems can be upgraded so vehicles can pick-up multiple packages and drop them off at once. Finally, a p-hub optimization algorithm can be used to determine the optimal depot location to minimize customer wait time, optimize charge time of the robots and drones, while maximizing companies profit. 


\section{Chapter 4}

\section{Additional Analysis}

In this chapter a further elaboration and an additional scenario analysis are added which were previously excluded from the journal article due to the word count restriction. 


\subsection{Additional Scenario}

An additional scenario of hybrid system utilizing both robots and drones, similar to scenario 3 is presented here. However, for this scenario the phases were switched. Here phase one will have drones pick-up the food packages from the restaurants and carry them back to the depot. Then phase two comes in, where the package is placed in the robot and delivered from the depot to its drop-off destination. This hybrid system will ease the process for customers, where no drop-off areas are required to be defined for each house. Furthermore, due to strict regulations regarding the commercial use of drones this system will reduce the number of drones flying in the air.

To differentiate between both hybrid scenarios, this study will refer to the first hybrid system with phase one being robots and phase two being drones as "Hybrid system 1.0" and for the additional hybrid scenario where phase one is carried out by drones and phase two is carried out by robots with "Hybrid System m 2.0"

\subsubsection{Proposed delivery process for hybrid delivery system}

This system utilizes both robots and drones together operating from a hub/depot. Both networks are used to operate this system and Intersections $I=\{\alpha, \beta, \mu\}$ can be identified as either Restaurant $(\beta)$, Home $(\alpha)$ or Depot $(\mu)$.

This is system is divided into two phases; phase one uses drones to go from the depot $(\mu)$ to pick up the food package at the restaurant $(\beta)$ and drops it off back at the depot $(\mu)$. The first phase was assigned to drones to reduce the fleet size of drones flying due to strict regulations. However, in this phase and due to the drones fast seed, packages are assigned for pick-up at least after 12 minutes from the time the request is placed to give sufficient time for the restaurant to prepare the food. Phase two uses robots to carry the food package from the depot $(\mu)$ to the home $(\alpha)$ and heads 
back to the depot $(\mu)$ empty. Phase two was chosen as robots to eliminate the need for a designated landing area to place the food. For this system one centralized dispatcher is created. However, two food request tables are generated from it; drone food request table $(\tau)$ and robot food request table $(\Upsilon)$

Once a food order (o) is placed by a costumer, the order (o) is placed in the drone food request table $(\tau)$. The dispatcher (Di) assigns an available drone to pick-u the package at least after 12 minutes from placing the order However, if the order has been in queue for more than 12 minutes it gets assigned as soon as a drone is available. The available drone (d_av) residing in the depot $(\mu)$ is assigned to pick-up the first package in queue from the restaurant $(\beta)$ and bring it back to the depot $(\mu)$. After dropping off the food package (p) at the depot the order is assigned to robot food request table $(\Upsilon)$ with a time stamp equivalent to the drone drop-off time of the package. When an available robot is located in the depot $(\mu)$, it gets reassigned to the first package in queue and delivers the (p) from the depot $(\mu)$ to the home $(\alpha)$ thus completing the delivery of the order (o). The details for the hybrid system are described in Algorithm 4 and the methodology is illustrated in Figures 14 and 15. 


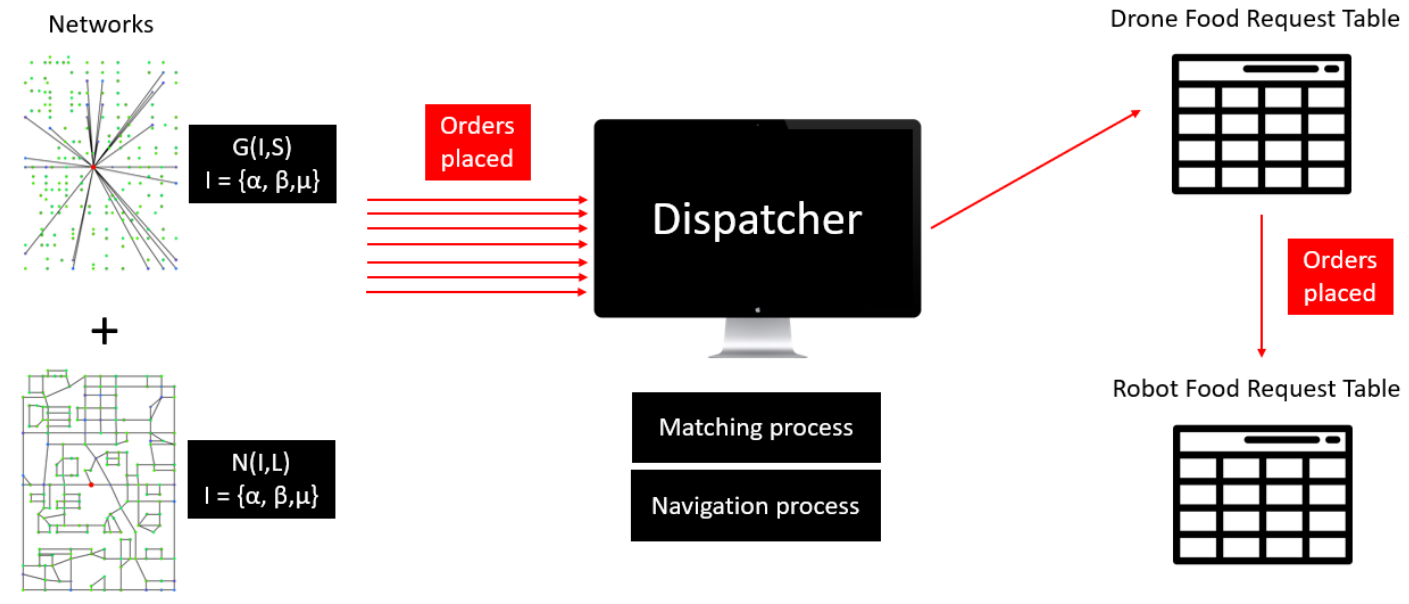

FIGURE 14 : Methodology of hybrid system 2.0

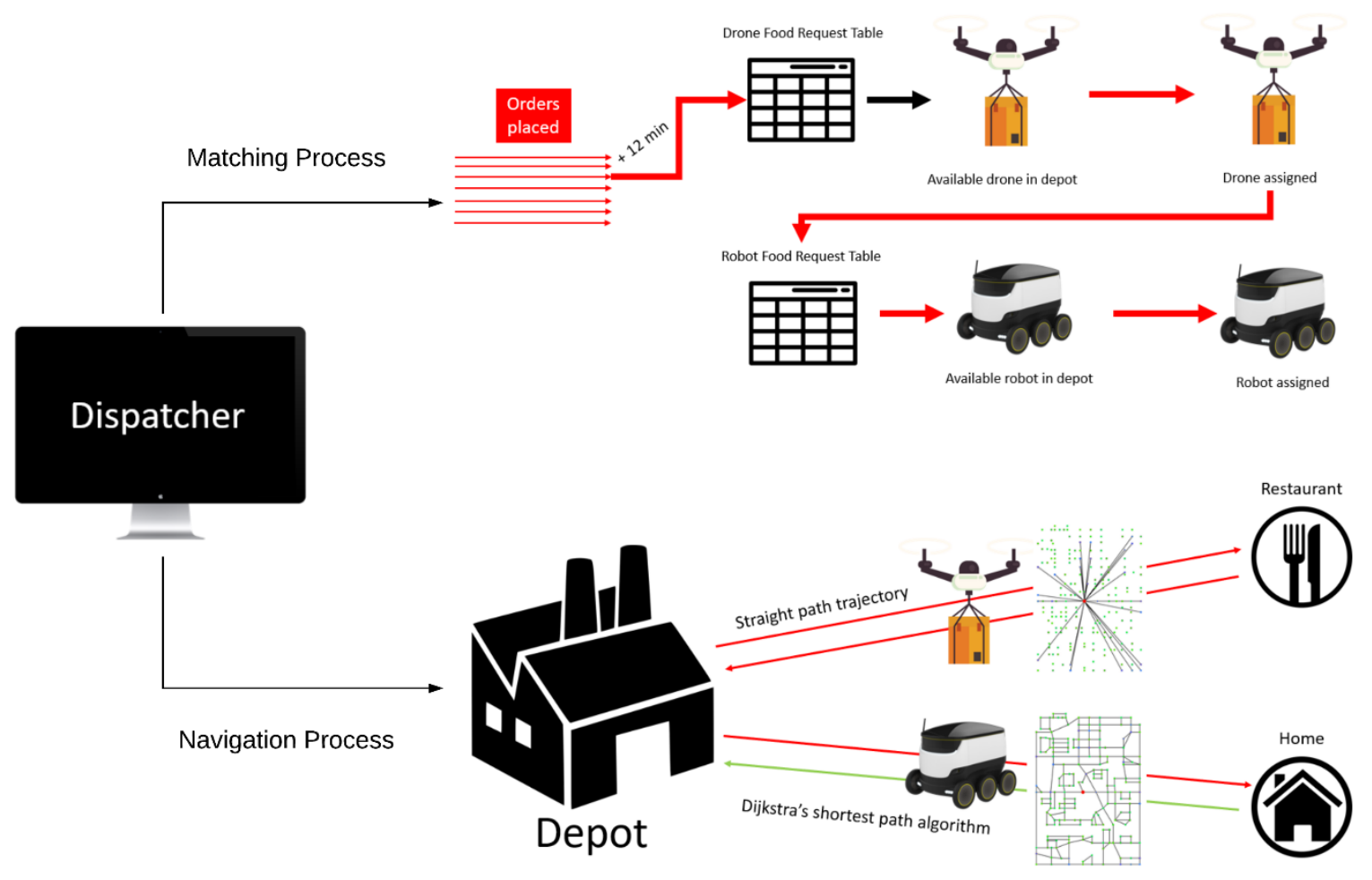

FIGURE 15 : Dispatcher processes for hybrid system 2.0 
1. $\mathrm{t}=1$;

2. Customer places order through the food delivery app;

3. Dispatcher (Di) identifies set of active requests $A o=\left\{\right.$ Ao_t $\left._{-}, \mathrm{Ao}_{-}\{\mathrm{t}+1\}, \ldots\right\}$;

4. while active requests (Ao) exist do

5. $\quad$ Dispatcher (Di) creates a drone food request table $(\tau)$ based on orders placed in chronological order;

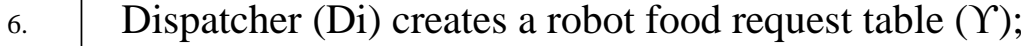

7. $\quad$ while $t$ - order request time $\left(\mathrm{R} \_t\right)>=12$ minutes;

8.

\section{if drone available (d_av) in depot $(\mu)$;}

Dispatcher (Di) assigns available drone (d_av) to the first active request lined up in queue (Ao_t) in $(\Upsilon)$;

(d) travels from depot $(\mu)$ to the assigned restaurant $(\beta)$ for pick-up;

(d) drops of (Ao_t) in depot $(\mu)$;

Dispatcher (Di) updates list of drones available(d_av) at depot $(\mu)$;

Dispatcher (Di) updates the set of orders (Ao_t) in $(\tau)$;

when (Ao_t) reaches depot $(\mu)$ do;

Dispatcher places (Ao_t) in queue in $(\Upsilon)$;

If robots available ( $\mathrm{r} \_$av) in $\operatorname{depot}(\mu)$;

Dispatcher (Di) assigns available robot (r_av) to the first active request lined up in queue (Ao_t) in $(\tau)$;

(r) travels from its location to the assigned home $(\alpha)$ for drop-off;

(r) drops-off (Ao_t) at home $(\alpha)$;

(r) returns to depot $(\mu)$;

Dispatcher (Di) updates list of robots available(r_av) at depot $(\mu)$;

Dispatcher (Di) updates the set of orders (Ao_t) in (Y);

else

Dispatcher (Di) waits until a robot becomes available in the depot $(\mu)$;

\section{end}

else

Dispatcher (Di) waits until a drone becomes available in depot $(\mu)$ to assign it to (Ao_t);

end

\section{else}

$t=t+1$

else

$t=t+1$;

end 


\subsubsection{Case Study}

The same area was used for the application of the fourth scenario with a total of 199 intersections and 286 streets links for robots phase shown in figure 16-b and 25 links for the drones phase shown in figure 16-a. In the simulation model nodes are identified as one of the following three; The red node represents the depot $(\mu)$ while the green nodes represent homes $(\alpha)$ and the blue represent restaurants $(\beta)$.

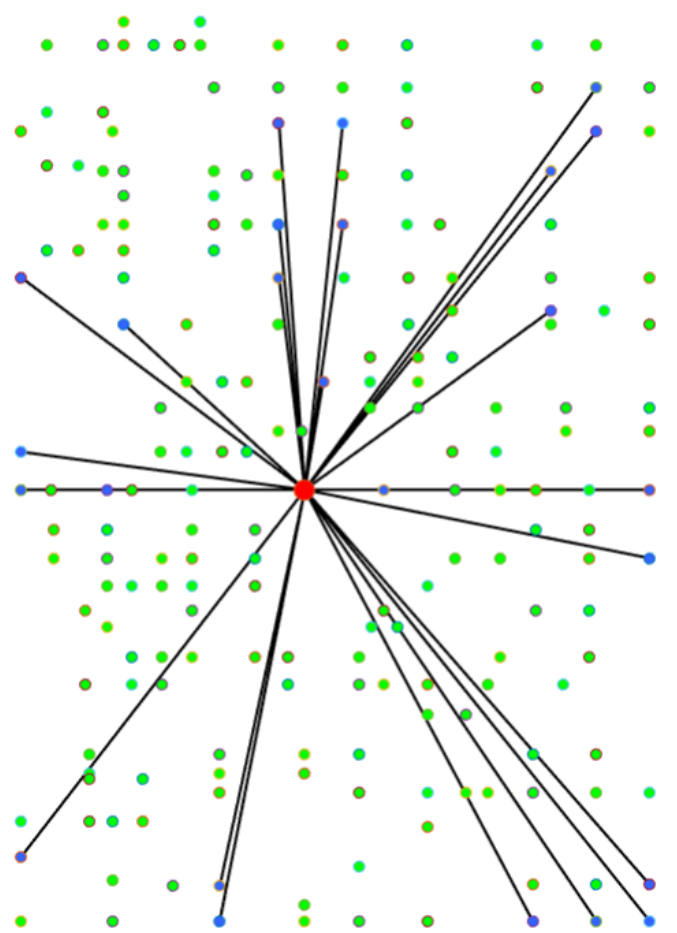

(a)

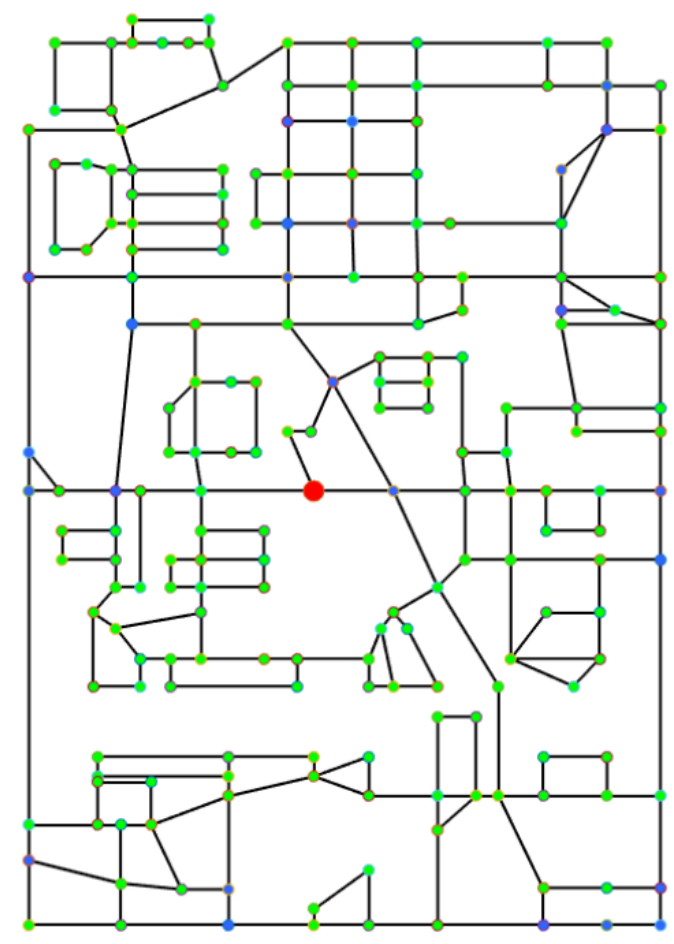

(b)

FIGURE 16 : Detailed network for scenario 4: (a) drone network - (b) robot network 
Furthermore, additional 12 scenarios were simulated and analyzed concluding the total number of scenarios to 30 scenarios for this case study as demonstrated in Table 5.

TABLE 5 List of Additional Scenarios

\begin{tabular}{|c|c|c|c|}
\hline Scenario number & System & Fleet Size & Demand \\
\hline 19 & Hybrid System & 10 drones - 20 robots & $20 \%$ increase \\
\hline 20 & Hybrid System & 10 drones - 25 robots & $20 \%$ increase \\
\hline 21 & Hybrid System & 10 drones - 30 robots & $20 \%$ increase \\
\hline 22 & Hybrid System & 15 drones - 20 robots & $20 \%$ increase \\
\hline 23 & Hybrid System & 15 drones - 25 robots & $20 \%$ increase \\
\hline 24 & Hybrid System & 15 drones - 30 robots & $20 \%$ increase \\
\hline 25 & Hybrid System & 20 drones - 20 robots & $20 \%$ increase \\
\hline 26 & Hybrid System & 20 drones - 25 robots & $20 \%$ increase \\
\hline 27 & Hybrid System & 20 drones - 30 robots & $20 \%$ increase \\
\hline 28 & Hybrid System & 25 drones - 20 robots & $20 \%$ increase \\
\hline 29 & Hybrid System & 25 drones - 25 robots & $20 \%$ increase \\
\hline 30 & Hybrid System & 25 drones - 30 robots & $20 \%$ increase \\
\hline
\end{tabular}




\subsubsection{Results \& Analysis}

Figure 17 presents the average wait time for the additional hybrid system scenarios, with phase one being drones and phase two being robots, 12 scenarios were analyzed in this system. It is evident that when the system is operated with 10 or 15 drones the change in fleet size of the robots doesn't affect the average delivery time of the system. For example, when we have 10 drones, whether or not we increase the fleet size of robots for phase two, the average wait time remains the same. This shows that the orders are waiting a long time in queue for the drones to come be picked-up. However, by increasing the drones fleet size from 10 to 15 drones for phase one the average wait time for the deliveries decreased by approximately $49 \%$. Therefore, the system was analyzed by increasing the fleet size of drone to 20 and 25 drones operating with various robots fleet sizes. When the fleet size of drones was increased the fluctuation of average wait time depending on the fleet size of robots began to show. 


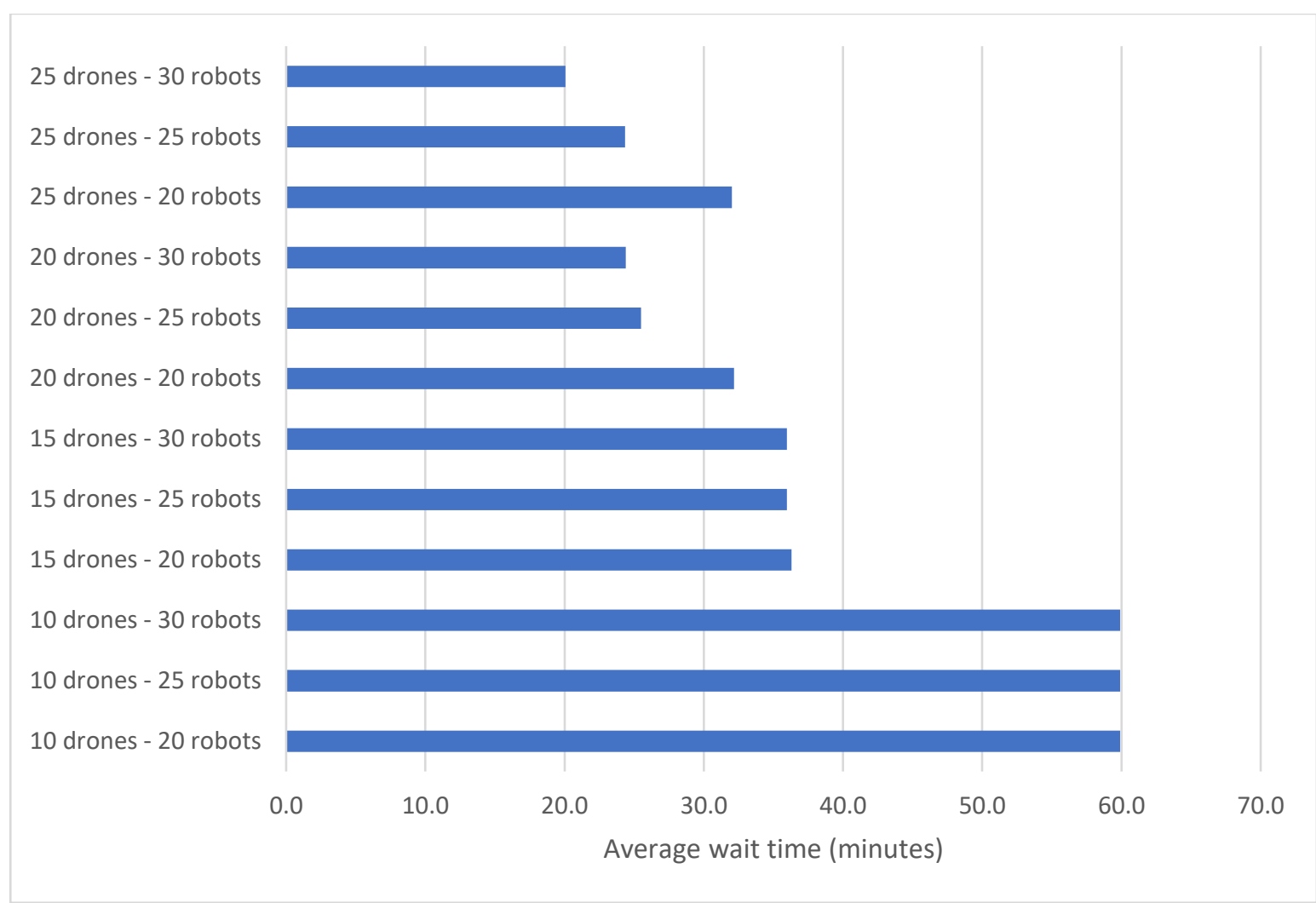

FIGURE 17 : Average wait time for hybrid system 2.0

While having an average wait time between (31-40) minutes is considered having an overall LOS $\mathrm{C}$, as mentioned in section 3.2.4 the performance of a system is weighed upon two criteria, the overall LOS of the system as well as the LOS for each order separately. Although the overall systems LOS is C. As shown in figure $18,8 \%$ of the orders have a LOS of F and $31 \%$ have a LOS of $\mathrm{D}$ which makes this scenario not efficient. The efficiency is based upon how long customers have to wait for their order to be delivered as per the performance criteria set in section 3.2.4. by referring to food delivery applications criteria for acceptable wait time. 


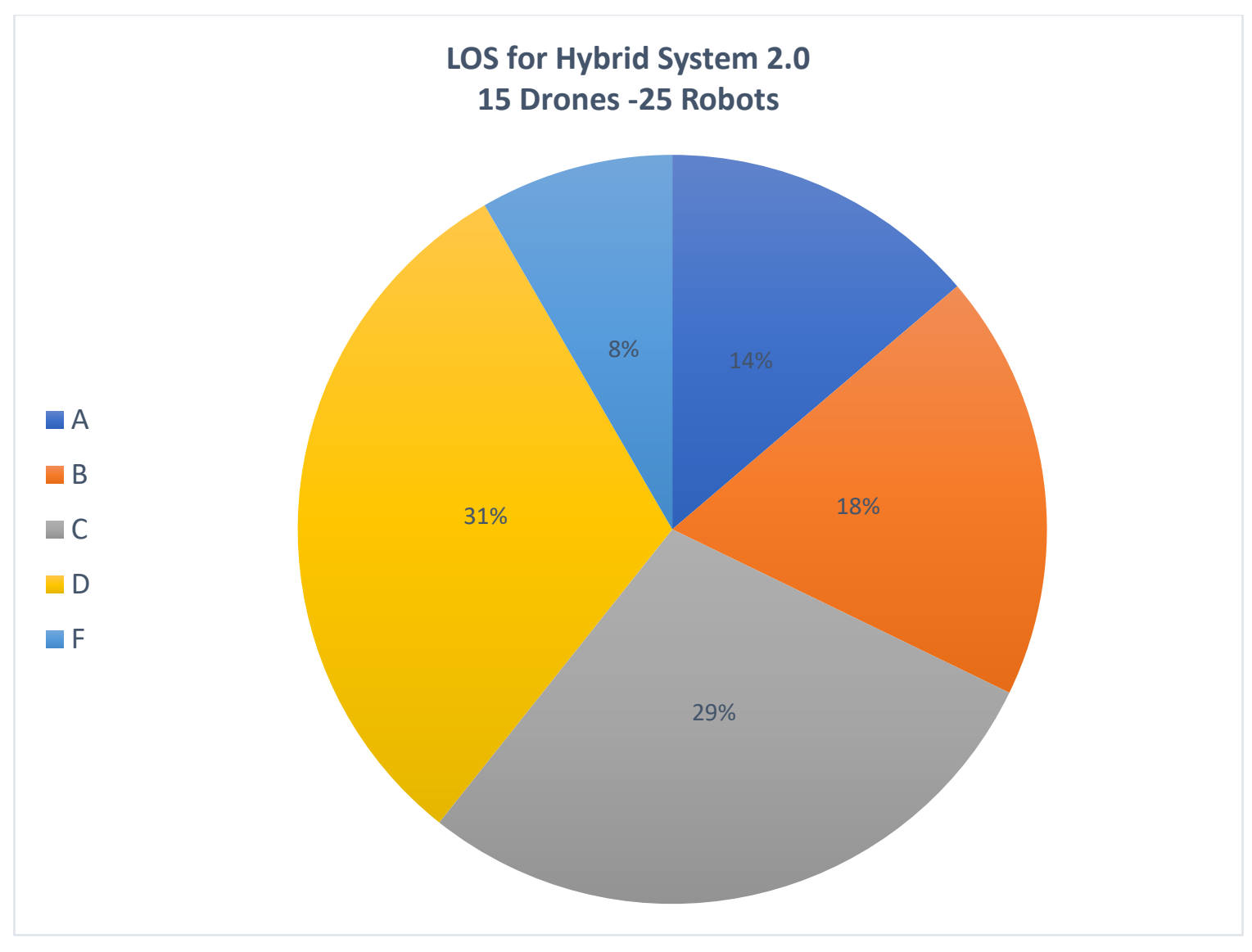

FIGURE 18 : LOS for hybrid system 2.0

When taking a closer look to the data, it is observed that the following scenarios give approximately the same average wait time for orders;

- 20 drones -25 robots

- 20 drones -30 robots

- 25 drones - 25 robots

- 25 drones -30 robots

To get a better understanding of these systems we plotted a boxplot chart with these scenarios as shown in in Figure 19. The least wait time among all scenarios is ( 25 drones - 30 robots) scenario with an average customer wait time of 20.1 minutes. However, the optimal starting fleet size won't be 25 drones - 30 robots due to one simple fact, why invest on buying extra fleet size while being 
able to invest in a smaller fleet size that can also deliver to customers within the acceptable performance criteria. The most efficient scenario that foresees the delivery for all the orders for the set of demand used is 20 drones and 25 robots with a maximum customer wait time of 34.4 minutes (LOS C) which is acceptable according to the performance criteria. The maximum wait time is less than $1 \%$ apart except for 25 drones- 30 robots scenario

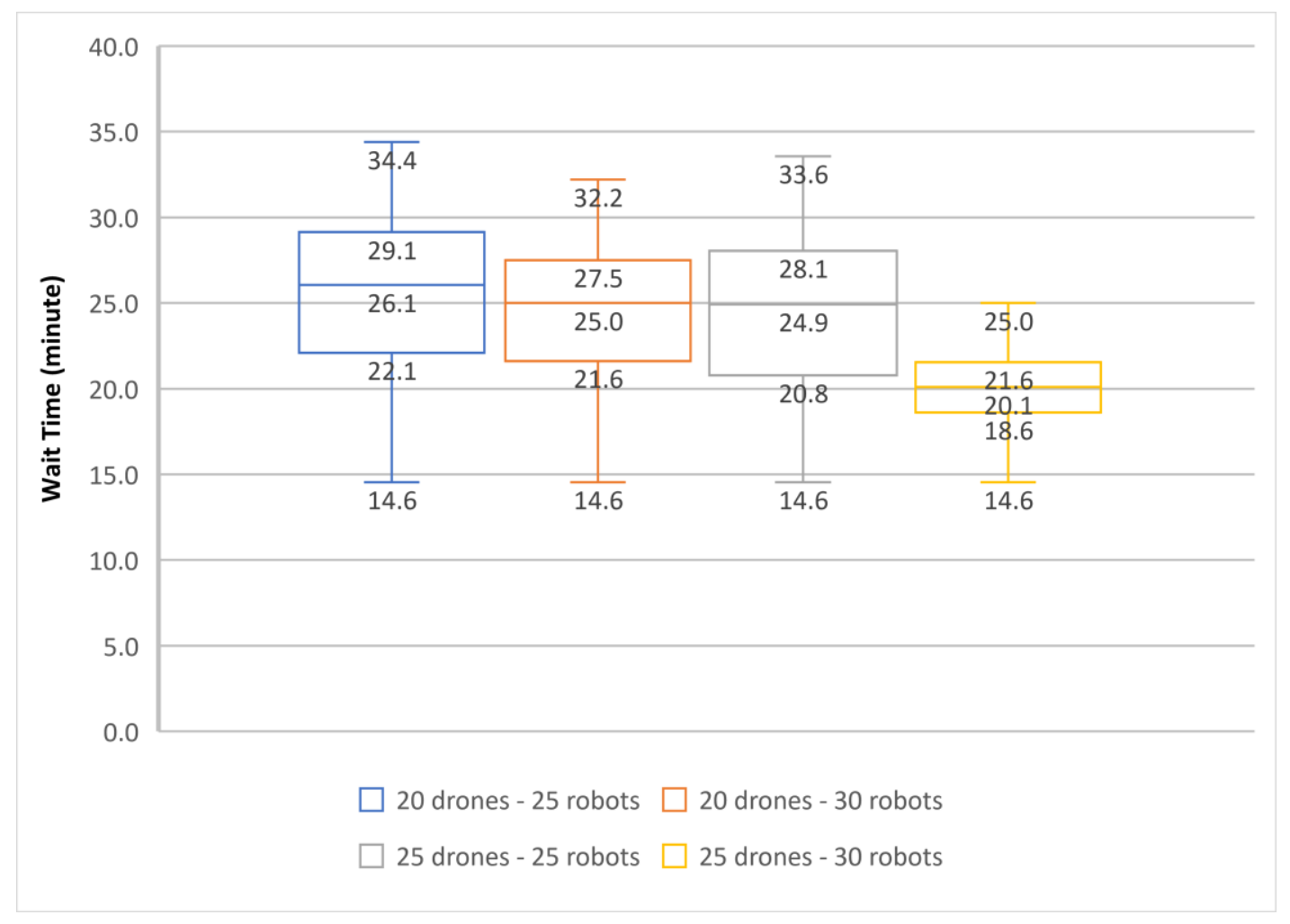

FIGURE 19 : Average wait time for optimal scenarios for hybrid system 2.0

In the two hybrid systems the delivery process is a static process meaning if the location of the drop-off is on the same path as phase one vehicle is operating and heading back to the depot the vehicle will not stop and drop off the food. The operation will determine that the vehicle used in phase one heads back to the depot drop of the food then the vehicle for phase two carries the food from the depot to the home destination for drop off. In our case this has happened, however the maximum wait time for the optimal starting fleet used for hybrid system 2.0 was approximately 
35 minutes which is an acceptable wait time however the system needs further improvement to make it more efficient and eliminate this problem by dropping off food that is on the same route as the vehicle heads back to the depot.

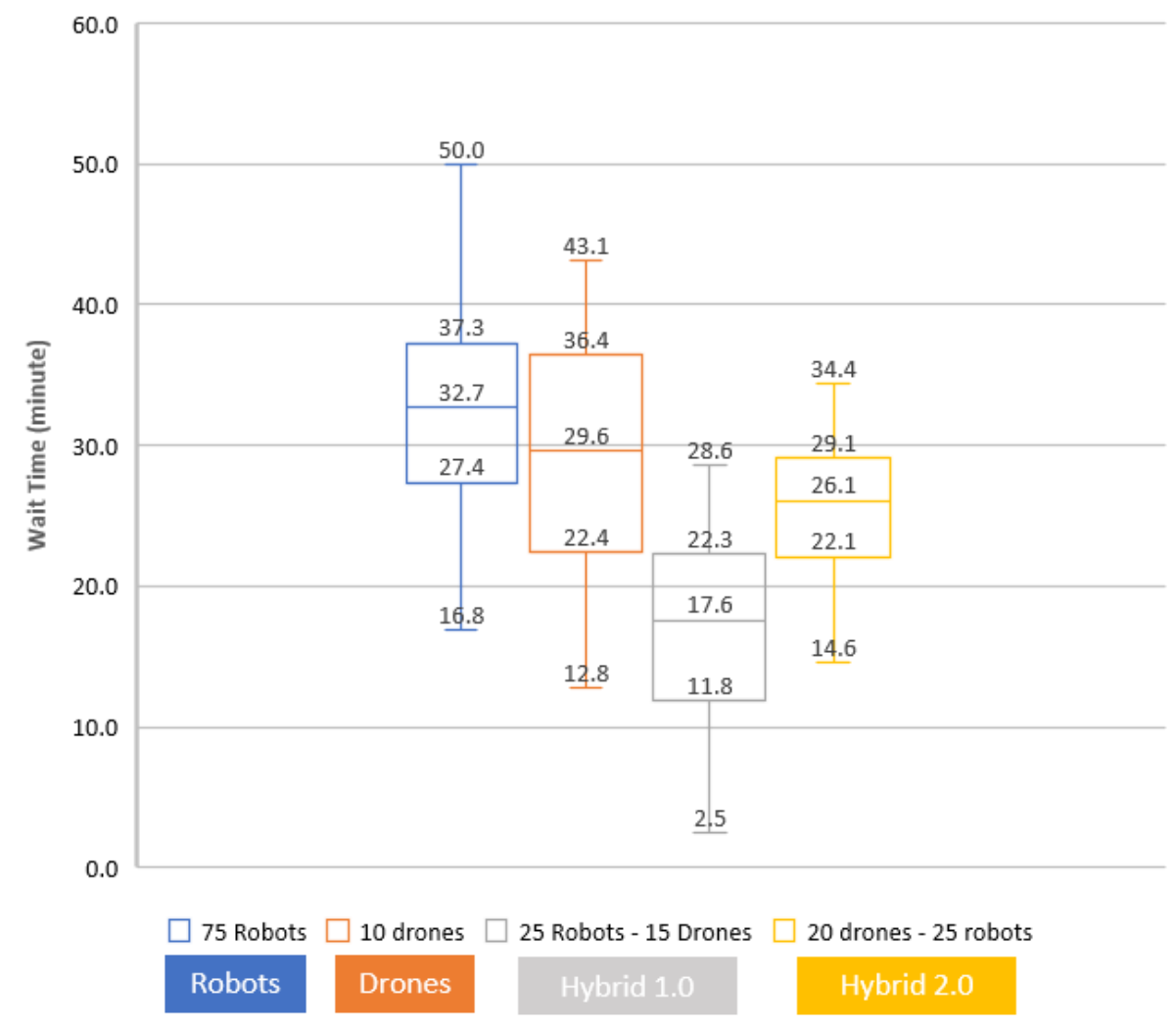

FIGURE 20 : Average wait time for optimal starting fleet sizes for all four systems

To compare all four systems designed a box plot of the average wait time for the optimal starting fleet sizes per system shown in figure 20 is used. For the robot and drone systems $50 \%$ of the orders are delivered above (30-33) minutes whereas both hybrid systems deliver in a relatively less wait time for customers. For example, hybrid system $2.0,50 \%$ of the orders are delivered more than 26 minutes and up to 35 minutes. 
Hybrid system 1.0 had an optimal starting fleet size of 25 robots and 15 drones which is in terms of total vehicle used less than Hybrid system 2.0 by 5 vehicles. Also, Hybrid system 1.0 reduces the maximum wait time from the robot system by $43 \%$ and from the drone system by $34 \%$ while Hybrid system 2.0 reduces the maximum wait time from the robot system by $31.2 \%$ and from the drone system by $20.1 \%$. That being said, Hybrid system 1.0 considered food preparation time accounted for by using robots for phase 1 but didn't take into consideration that the distance of restaurant nodes located closer to the depot take less than 12 minutes for the drones to reach. Furthermore, having drones deliver to homes have its certain regulations and concerns. Every house must have a designated landing area for drones and the public tends to have security concerns regarding packages landing from the sky. Therefore, we believe the most efficient and applicable system is Hybrid system 2.0, where we need in our area of study 25 designated landing areas at restaurants and more control over drones flying routes.

With the use of autonomous vehicles on demand food delivery companies will be owning the fleet unlike the existing systems whereas car owners use their personal vehicles to deliver food. In the case, vehicle storage, charging and maintenance lies on the owning company. If a vehicle breaks down will be directly affected so it is vital to maintain vehicles throughout the year and especially in harsh weather conditions. Owning extra vehicles will ensure that the operation stays on track and the average wait time per customer will not be affected. 


\section{Chapter 5}

\section{Concluding Remarks}




\subsection{Conclusion}

This dissertation presents the design and application of last-mile on-demand food delivery systems using autonomous technologies such as drones and robots. Four systems were designed, Robot System, Drones System, Hybrid System 1.0 and Hybrid System 2.0. Level of Service (LOS) criteria that was used to evaluate the efficiency of these systems was based upon customer waiting time only. LOS criteria can expand to cost of delivery, energy consumption, Green House Gas Emissions and vehicle Kilometer travelled per vehicle.

Each technology varies in shape, size, manufacturing company, model, battery, motor, sensors charging systems and application platform. With all these differences, capital and operational will vary accordingly and if Hybrid system 1.0 or 2.0 were to be applied a hub needs to be built. However, large companies such as Amazon, Google, Doordash, etc. are moving towards integrating these technologies into their current delivery systems implies that these technologies carry benefits in terms of cost. The first system designed and evaluated was the robots only system. This system primary advantage is the robot's ability to navigate the sidewalk without using the roads. Nevertheless, in order to obtain an acceptable systems performance a large fleet size was needed making it the least economical system among all four systems.

The second system is the drones only system. Drones have an advantage of flying in straight lines thus not needing to follow a road network in significantly faster speeds and dramatically fewer fleet sizes than robots. Yet there are strict rules and regulations in terms of the use of drones for commercial use also, safety concerns of drones navigating areas with high rise buildings or drones crashing in the air hold back the deployment of these drones. 
Consequently, in order to gain the advantages and capabilities of both technologies Hybrid system 1.0 and 2.0 were introduced and a hub was located in our network as an operating, maintenance, charging and storage center for all the vehicles. Hybrid system 1.0 used robots for phase one of the delivery to pick up orders from restaurants and carry them back to the hub where phase two was performed by drones to take the food from the hub and drop it off to the homes. In Hybrid system we used drones to preform phase one and robots to preform phase two while adding a mandatory food preparation time of 12 minutes to each order before getting picked up by the drone in order to account for the drones high speed and trajectory which can be considered a disadvantage if the drone arrives early.

To test the design of these systems, an area of approximately $5.80 \mathrm{~km}^{2}$ was chosen in the city of Mississauga. The area had no highways since the robots operate via sidewalks, also we assumed all high-rise buildings as flat terrain for the drones to operate via straight distances. However, some elements regarding the design of these systems need to be modified per city to be applicable. For example, City of Toronto, especially downtown core, is mostly high-rise building restricting the application of drones. Therefore, the design of food delivery systems needs to be adjusted based upon the topography and characteristic of the city nevertheless after adjustments these systems can be applicable and fully integrated into the food delivery system.

Every system designed has its own set of advantages and drawbacks but Hybrid system 2.0 is believed to be the most feasible and operational system of all four since the advantages of the drones counterbalance the disadvantages of robots and vise-versa thus leading to a fully integrated last-mile on-demand food delivery system. 


\subsection{Key recommendations for application}

For the application of last mile on demand food delivery systems in The City of Mississauga, following recommendations are suggested:

1- Rules and regulations need to be developed/updated for the implementation of these autonomous technologies. This framework should seek to reduce traffic congestion, increase safety levels, reduce GHG and sustain economic vitality.

2- data and insights should be provided to the universities and companies for research and innovation regarding these autonomous technologies, interactions with humans, safety and performance requirements and how they perform in cold, snowy weather.

3- Sidewalks and dedicated lanes need to be maintained constantly to ensure the robots preform to their best capability.

4- City planners, transportation planners and engineers need to collaborate to enable the transition from a fully car driven last mile delivery systems to an integrated autonomous delivery system.

5- Ways of expanding these systems to wider areas and regions need to be taken into consideration to accommodate the entire city and reach as many consumers.

These systems can scale-up beyond the area chosen in our case study by adding more than one hub location, increasing fleet size and using drones to navigate certain areas that have limited access due to non-existing sidewalks.

\subsection{Future work}

For each system designed there is room for improvement. For future work the following aspects can be considered:

1- The separation of orders based on weight of package and integrating the use of autonomous vehicles for orders with heavy weight (catering orders). 
2- In this dissertation the orders were matched with vehicles based on First-in First-out principle (FIFO), where every vehicle carries one package only. For future purposes a sophisticated algorithm can be developed to match vehicles to more than one order based on the weight and space left in the vehicle.

3- The hub location was assigned based on a strategic location however optimization can be used to determine the optimal hub location based on a set of criteria such as cost, distance traveled, energy and greenhouse gas emissions.

4- For the full deployment of this system an analysis can be conducted to calculate the amount of reduction in greenhouse gas emissions occurs with the use of these automated technologies.

5- Our hybrid systems are static systems, for future purposes we can investigate the possibility of turning these systems into dynamic systems where there aren't fixed phases but the type of vehicle or using two vehicles will be assigned based on the orders specifications such as taking into consideration the location of the restaurant, home and depot.

These emerging disruptive technologies are considered new and unprecedented. To the best of our knowledge there has been no fully operable system designed and evaluated for last-mile ondemand system using these emerging technologies. This thesis serves as an initial step to the integration and deployment of these technologies in our current food delivery systems as they will initially complement and not replace the exciting systems that exist. 


\section{References}

[1] "Urban freight/city logistics | Eltis." [Online]. Available: https://www.eltis.org/topics/urban-freightcity-logistics. [Accessed: 19-Aug-2019].

[2] "Urban freight distribution," Wikipedia. 28-Mar-2019.

[3] "68\% of the world population projected to live in urban areas by 2050, says UN," UN DESA | United Nations Department of Economic and Social Affairs, 16-May-2018. [Online]. Available: https://www.un.org/development/desa/en/news/population/2018revision-of-world-urbanization-prospects.html. [Accessed: 20-Aug-2019].

[4] I. Cardenas Barbosa, Y. Borbon-Galvez, T. Verlinden, E. Van de Voorde, T. Vanelslander, and W. Dewulf, "City logistics, urban goods distribution and last mile delivery and collection," Compet. Regul. Netw. Ind., vol. 18, p. 178359171773650 , Nov. 2017.

[5] "With online shopping on the rise, cities look to address congestion impacts of deliveries," Mobility Lab, 13-Apr-2017. [Online]. Available: https://mobilitylab.org/2017/04/13/role-ofdeliveries-in-congestion/. [Accessed: 07-Aug-2019].

[6] "Freight and Congestion - FHWA Freight Management and Operations." [Online]. Available: https://ops.fhwa.dot.gov/freight/freight_analysis/freight_story/congestion.htm. [Accessed: 06-Aug-2019].

[7] T. Canada, "Transportation in Canada 2016." 2016.

[8] J.-F. Cordeau, G. Laporte, J.-Y. Potvin, and M. W. P. Savelsbergh, "Chapter 7 Transportation on Demand," in Handbooks in Operations Research and Management Science, vol. 14, Elsevier, 2007, pp. 429-466.

[9] “TNC VMT Findings_Memo 08.01.2019.pdf," Google Docs. [Online]. Available: https://drive.google.com/file/d/1FIUskVkj9lsAnWJQ6kLhAhNoVLjfFdx3/view?usp=embe d_facebook. [Accessed: 07-Aug-2019].

[10] "Online Food Delivery - Canada | Statista Market Forecast," Statista. [Online]. Available: https://www.statista.com/outlook/374/108/online-food-delivery/canada. [Accessed: 08Aug-2019].

[11] "Uber Eats plans to expand in Canada beyond restaurant delivery | CTV News." [Online]. Available: https://www.ctvnews.ca/business/uber-eats-plans-to-expand-in-canada-beyondrestaurant-delivery-1.4271007. [Accessed: 07-Aug-2019].

[12] "Peel Region Launches Off-Peak Delivery Pilot - Ontario Trucking Association.".

[13] "Doddle Parcels | Parcel Collections and Return Service." [Online]. Available: https://doddle.com/. [Accessed: 07-Aug-2019].

[14] "Final 50 Feet Research Program | Supply Chain Transportation and Logistics Center at the University of Washington." [Online]. Available:

http://depts.washington.edu/sctlctr/research-project-highlights/urban-goods-delivery-0. [Accessed: 07-Aug-2019].

[15] "Worldwide Retail Ecommerce Sales: The eMarketer Forecast for 2016 - eMarketer." [Online]. Available: https://www.emarketer.com/Report/Worldwide-Retail-EcommerceSales-eMarketer-Forecast-2016/2001849. [Accessed: 08-Jan-2019].

[16] J. Visser, T. Nemoto, and M. Browne, "Home Delivery and the Impacts on Urban Freight Transport: A Review," Procedia - Soc. Behav. Sci., vol. 125, pp. 15-27, Mar. 2014.

[17] T. R. Leinbach and C. Capineri, Eds., Globalized freight transport: intermodality, ecommerce, logistics and sustainability. Cheltenham, UK ; Northampton, MA: Edward Elgar, 2007. 
[18] "What is Last Mile Delivery? Part One," Datex Corporation, 18-Feb-2016. .

[19] B. Allen, "Improving freight efficiency within the 'last mile': A Case study of Wellington's Central Business District," Thesis, University of Otago, 2012.

[20] "Urban area (UA)." [Online]. Available: https://www12.statcan.gc.ca/censusrecensement/2006/ref/dict/geo049-eng.cfm. [Accessed: 29-Dec-2018].

[21] K. J. Stefan, J. D. P. McMillan, and J. D. Hunt, "Urban Commercial Vehicle Movement Model for Calgary, Alberta, Canada," Transp. Res. Rec., p. 10, 1921.

[22] M. Ferguson, H. Maoh, T. H. Rashidi, J. Ryan, and P. Kanaroglou, "Estimating Urban Commercial Vehicle Movements in the Greater Toronto-Hamilton Area," p. 29.

[23] W. Qi, L. Li, S. Liu, and Z.-J. M. Shen, "Shared Mobility for Last-Mile Delivery: Design, Operational Prescriptions, and Environmental Impact," Manuf. Serv. Oper. Manag., vol. 20, no. 4, pp. 737-751, Sep. 2018.

[24] S. Iwan, K. Kijewska, and J. Lemke, “Analysis of Parcel Lockers' Efficiency as the Last Mile Delivery Solution - The Results of the Research in Poland," Transp. Res. Procedia, vol. 12, pp. 644-655, Jan. 2016.

[25] "On-demand Transport: A discussion paper for future innovation," www.transport.wa.gov.au.

[26] K. Dorling, J. Heinrichs, G. G. Messier, and S. Magierowski, "Vehicle Routing Problems for Drone Delivery,” IEEE Trans. Syst. Man Cybern. Syst., vol. 47, no. 1, pp. 70-85, Jan. 2017.

[27] Q. M. Ha, Y. Deville, Q. D. Pham, and M. H. Hà, "On the min-cost Traveling Salesman Problem with Drone,” Transp. Res. Part C Emerg. Technol., vol. 86, pp. 597-621, Jan. 2018.

[28] C. C. Murray and A. G. Chu, "The flying sidekick traveling salesman problem: Optimization of drone-assisted parcel delivery," Transp. Res. Part C Emerg. Technol., vol. 54, pp. 86-109, May 2015.

[29] S. M. Ferrandez, T. Harbison, T. Weber, R. Sturges, and R. Rich, "Optimization of a truckdrone in tandem delivery network using k-means and genetic algorithm," J. Ind. Eng. Manag. Barc., vol. 9, no. 2, pp. 374-388, 2016.

[30] Z. Yuan and Y. Y. Gong, "Bot-In-Time Delivery for Robotic Mobile Fulfillment Systems," IEEE Trans. Eng. Manag., vol. 64, no. 1, pp. 83-93, Feb. 2017.

[31] S. C. Government of Canada, "The Daily - Vehicle registrations, 2016," 29-Jun-2017. [Online]. Available: https://www150.statcan.gc.ca/n1/daily-quotidien/170629/dq170629deng.htm. [Accessed: 04-Jul-2019].

[32] S. C. Government of Canada, "The Daily - Vehicle registrations, 2017," 15-Jun-2018. [Online]. Available: https://www150.statcan.gc.ca/n1/daily-quotidien/180615/dq180615eeng.htm. [Accessed: 04-Jul-2019].

[33] Castiglione, Joe, Cooper, Drew, Sana, Bhargava, Tischler, Dan, and Chang, Tilly, "TNCs \& Congestion." Oct-2018.

[34] Joerss , Martin, Schröder , Jürgen, F. Neuhaus, Klink , Christoph, and Mann, Florian, "Parcel delivery the future of last mile." McKinsey\&Company, Sep-2016.

[35] T. Hoffmann and G. Prause, "On the Regulatory Framework for Last-Mile Delivery Robots," Machines, vol. 6, no. 3, p. 33, Sep. 2018.

[36] "parcel_delivery_the_future_of_last_mile.pdf." .

[37] Jung, Sunghun and kim hyunsu, "Analysis of Amazon Prime Air UAV Delivery Service," J. Knowl. Inf. Technol. Syst., vol. 12, no. 2, pp. 253-266, Apr. 2017. 
[38] A. Goodchild and J. Toy, "Delivery by drone: An evaluation of unmanned aerial vehicle technology in reducing CO2 emissions in the delivery service industry," Transp. Res. Part Transp. Environ., vol. 61, pp. 58-67, Jun. 2018.

[39] S. Abdinnour-Helm, "Network design in supply chain management," Int. J. Agile Manag. Syst., Aug. 1999.

[40] J. Jung and R. Jayakrishnan, "High-Coverage Point-to-Point Transit: Electric Vehicle Operations," Transp. Res. Rec. J. Transp. Res. Board, vol. 2287, no. 1, pp. 44-53, Jan. 2012.

[41] S. C. Government of Canada, "Census Profile, 2016 Census," 08-Feb-2017. [Online]. Available: https://www12.statcan.gc.ca/census-recensement/2016/dp$\mathrm{pd} /$ prof/index.cfm?Lang=E. [Accessed: 24-Jul-2019]. 